\title{
The evolution of the Puf superfamily of proteins across the tree of eukaryotes
}

\author{
Vladimíra Najdrová1, Courtney W. Stairs², Martina Vinopalová', Luboš Voleman and Pavel Doležal ${ }^{1 *}$ (D)
}

\begin{abstract}
Background: Eukaryotic gene expression is controlled by a number of RNA-binding proteins (RBP), such as the proteins from the Puf (Pumilio and FBF) superfamily (PufSF). These proteins bind to RNA via multiple Puf repeat domains, each of which specifically recognizes a single RNA base. Recently, three diversified PufSF proteins have been described in model organisms, each of which is responsible for the maturation of ribosomal RNA or the translational regulation of mRNAs; however, less is known about the role of these proteins across eukaryotic diversity.
\end{abstract}

Results: Here, we investigated the distribution and function of PufSF RBPs in the tree of eukaryotes. We determined that the following PufSF proteins are universally conserved across eukaryotes and can be broadly classified into three groups: (i) Nop9 orthologues, which participate in the nucleolar processing of immature 18S rRNA; (ii) 'classical' Pufs, which control the translation of mRNA; and (iii) PUM3 orthologues, which are involved in the maturation of 75 rRNA. In nearly all eukaryotes, the rRNA maturation proteins, Nop9 and PUM3, are retained as a single copy, while mRNA effectors ('classical' Pufs) underwent multiple lineage-specific expansions. We propose that the variation in number of 'classical' Pufs relates to the size of the transcriptome and thus the potential mRNA targets. We further distinguished full set of PufSF proteins in divergent metamonad Giardia intestinalis and initiated their cellular and biochemical characterization.

Conclusions: Our data suggest that the last eukaryotic common ancestor (LECA) already contained all three types of PufSF proteins and that 'classical' Pufs then underwent lineage-specific expansions.

Keywords: RNA-binding protein, RNA processing, LECA, Puf superfamily proteins, Giardia intestinalis

\section{Background}

The Puf superfamily (PufSF) of proteins encompasses a class of eukaryotic RNA-binding proteins (RBPs), which interact with the 3 '-untranslated regions (3'-UTRs) of mRNA in the cytosol or with the precursors of rRNA molecules in the nucleolus [1-3]. The name of the protein family is derived from Pumilio and Fem-3 binding factor of Drosophila melanogaster and Caenorhabditis elegans, respectively $[4,5]$. PufSF proteins are defined by the presence of Puf repeats, each of which binds a single base of

\footnotetext{
* Correspondence: pavel.dolezal@natur.cuni.cz

'Department of Parasitology, Faculty of Science, Charles University, BIOCEV, Průmyslová 595, 25250 Vestec, Czech Republic

Full list of author information is available at the end of the article
}

an RNA molecule [6-9]. In the past two decades, three types of PufSF proteins (i.e. Puf, Nop9, and PUM3, discussed below) have been distinguished according to their biological role and structural arrangement [2, 10-12].

The so-called classical Puf proteins (Pufs) bind 3'UTRs of mRNA and usually have eight Puf repeats that organize into a crescent-shaped structure [13]. Each Puf repeat contains a tripartite recognition motif (TRM), and it is the combinations of eight TRMs, which specifies the sequence motif at which a particular Puf binds. In vivo, a single Puf protein can recognize hundreds of transcripts and, together with additional protein partners, regulate protein translation [1]. Puf proteins can mediate the repression [14-16], the activation [17] of

(c) The Author(s). 2020 Open Access This article is licensed under a Creative Commons Attribution 4.0 International License, which permits use, sharing, adaptation, distribution and reproduction in any medium or format, as long as you give appropriate credit to the original author(s) and the source, provide a link to the Creative Commons licence, and indicate if changes were made. The images or other third party material in this article are included in the article's Creative Commons licence, unless indicated otherwise in a credit line to the material. If material is not included in the article's Creative Commons licence and your intended use is not permitted by statutory regulation or exceeds the permitted use, you will need to obtain permission directly from the copyright holder. To view a copy of this licence, visit http://creativecommons.org/licenses/by/4.0/. The Creative Commons Public Domain Dedication waiver (http://creativecommons.org/publicdomain/zero/1.0/) applies to the data made available in this article, unless otherwise stated in a credit line to the data. 
gene expression, or the site-specific translation $[18,19]$. These proteins function in the cytoplasm.

The two other types of PufSF proteins operate predominantly within the nucleolus, where ribosome biogenesis takes place [20]. Nop9 proteins carry 11 Puf repeats organized into a U-shaped structure, which participate in the processing and folding of $18 \mathrm{~S}$ rRNA in the nucleolus $[10,21,22]$, while PUM3 proteins (including the human PUM3/PufA and yeast Puf6) bind double-stranded DNA or RNA without a sequence specificity [2, 23]. Similar to Nop9, PUM3 proteins carry 11 Puf repeats, although organized in L-shaped domain, and they are involved in the nucleolar processing of the large subunit of the ribosome [23], potentially during the processing of $7 \mathrm{~S}$ to $5.8 \mathrm{~S}$ rRNA [2].

Although the PufSF proteins are essential for core processes of the eukaryotic cell, such as rRNA maturation, investigations of PufSF proteins have been restricted to only a few lineages of eukaryotes (e.g. Opisthokonta and Viridiplantae). These studies recognized that the number of PufSF proteins within and between lineages of eukaryotes is highly variable $[24,25]$. Due to the number of different splice isoforms and the repetitive nature of the proteins, it has been difficult to delineate the evolutionary history of this essential protein family throughout eukaryotic evolution. Moreover, given the role of PufSF proteins in regulating the translation of a complex network of transcripts in modern eukaryotes $[19,26]$, it is tempting to speculate that PufSF proteins played a role in the origin of cellular complexity in eukaryotes. Indeed, such information is critical to understand the true nature of the last eukaryotic common ancestor (LECA); a concept of which can be drawn upon by examining the cellular properties of diverse eukaryotes, including the highly diverged organisms [27].

Metamonada represent highly diverged unicellular anaerobic eukaryotes [28] carrying specialized mitochondria [29]. Some Metamonada are important human and animal pathogens [30]. One of the best studied species of Metamonada is the human intestinal parasite Giardia intestinalis. Although the RNA metabolism in G. intestinalis is poorly understood, we would argue that this organism can be useful in studying various aspects of eukaryotic RNA biology owing to its transcriptome streamlining and overall extreme biology. For example, unlike most eukaryotes, the processing of rRNA and the actual nature of G. intestinalis nucleolus are still under debate [31]. Moreover, G. intestinalis generates large number of sterile transcripts of unknown function, which are both capped and polyadenylated [32]. To date, only six cis-spliced and two trans-spliced transcripts have been described in G. intestinalis making it easier to predict the transcriptome purely from genomic data [33, 34]. The 5'-untranslated regions (5'-UTRs) of G. intestinalis mRNAs are efficiently capped and bound by the ribosome despite being extremely short (i.e. 0-14 nucleotides) [35, 36]. Therefore, posttranscriptional regulation of gene expression is mostly limited to the stability and sequestration of the mRNAs [37]. Thus, 3'UTRs remain the key regions of mRNAs, which affect its stability and localization via the interaction with RNAbinding proteins [37].

Here, we report systematic bioinformatic survey of distribution of PufSF proteins with sampling across major eukaryotic supergroups. Our analyses show three groups of proteins encompassing (i) Nop9, (ii) Puf, and (iii) PUM3 homologues. In a given organism, Nop9 and PUM3 are usually represented by a single gene, while the number of Pufs is highly variable. However, the actual number of Pufs correlates with the number of transcripts of the particular lineages and thus the number of putative mRNA targets. These data also suggest that the LECA already contained one Nop9, one PUM3, and two Puf proteins and that the large copy number of Pufs in modern organisms can be explain by lineage-specific expansions.

We were able to identify all three PufSF proteins even within G. intestinalis. However, their initial characterization points to unique adaptations in G. intestinalis RNA metabolism.

\section{Results \\ Classification of PufSF proteins}

We initially classified the PufSF proteins across eukaryotic diversity. First, we performed a clustering analysis based on sequence similarity. This unbiased approach is based on mutual pairwise BLAST comparisons, and it is especially useful for the analysis of large protein datasets [38]. The initial dataset contained 4469 unique eukaryotic proteins carrying Puf repeat domain(s) (PF00806). No PufSF proteins were identified in Archaea or Bacteria, confirming the eukaryotic origin of the family. The clustering analysis revealed three major groups of PufSF proteins (Fig. 1, Additional file 1: Table S1): the Puf cluster, consisting of 2955 proteins containing eight Puf repeats in the C-terminal part of the protein; the PUM3 cluster, consisting of 674 PUM3 orthologues, including plant PUM24 proteins, with up to eleven Puf repeats; and the Nop9 cluster, consisting of 675 Nop9 proteins including the plant PUM23 proteins.

The proximity of the Puf and PUM3 proteins in our clustering analysis suggests that these proteins are more similar at the sequence level when compared to Nop9s, despite carrying different numbers of Puf repeats. Given the conservation of 11 Puf repeats in both the rRNAbinding proteins PUM3 and Nop9 proteins, we suspect that this was the ancestral domain arrangement of all members of the PufSF. Following adaptation to 


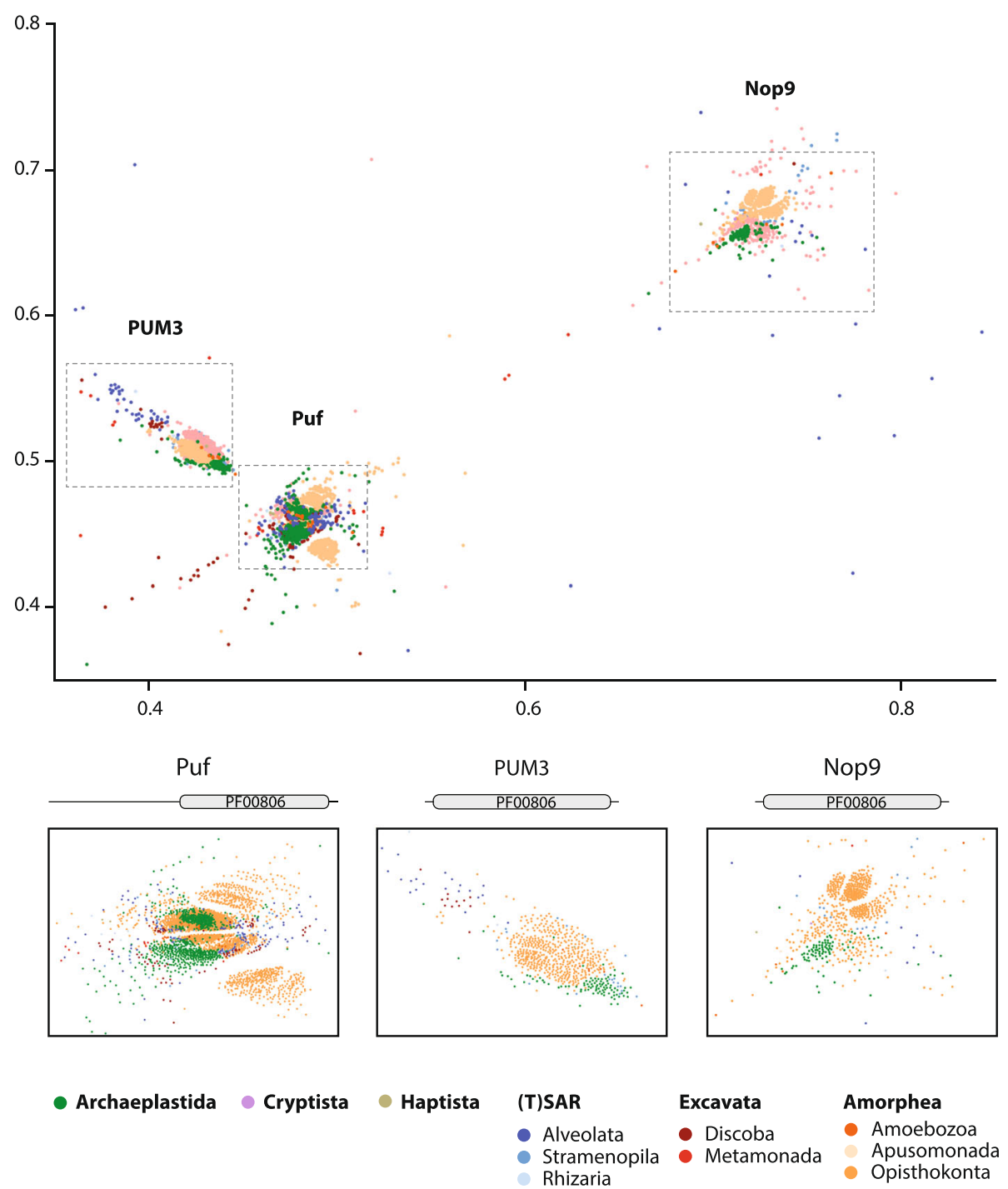

Fig. 1 Clustering analysis of Puf family proteins. PufSF proteins were analysed by CLANS. A total of 4469 proteins formed three major clusters of proteins corresponding to Nop9 homologues (Nop9 cluster), 'classical' Puf proteins (Puf cluster), and PUM3 orthologues (PUM3 cluster). The protein sequences were colour-coded according to the taxonomic affiliation to the eukaryotic supergroup. The details of each large cluster show mostly lineage-specific subclusters, except two large subclusters of Puf proteins. The diagrams above the cluster details depict the general domain arrangement of PufSF proteins; PF00806 corresponds to Puf RNA-binding repeat. The $x$ and $y$ axes represent relative positions of the protein sequence in 2D CLANS

interaction with mRNA molecules, the Puf proteins adopted a domain arrangement of only eight Puf domains $[2,21,22]$. The clustering approach was not sensitive enough to reveal detailed taxon-specific grouping of PufSF proteins except for the most represented eukaryotic groups of animals, plants, and fungi. The only exception was the formation of two clear large subclusters within the Puf cluster (Fig. 1) containing proteins of mixed taxonomic affiliation, which indicated the existence of at least two different Puf orthologues in the last common ancestor of eukaryotes. The position of eukaryotic supergroups encompassing protist lineages was rather dispersed across the subclusters. Metamonad proteins including the G. intestinalis sequences represented one of the most diverge proteins of the family.

\section{Taxonomic distribution of PufSF proteins}

The number of PufSF proteins encoded in a given genome differs significantly across the eukaryotic diversity (e.g. [4, 25]). Hence, we surveyed the distribution of PufSF of proteins on a species level across the tree of eukaryotes. To this aim, we retrieved all eukaryotic 1180 reference proteomes from UniProtKB and classified them as Puf, PUM3, or Nop9 orthologues. While the 
dataset is biassed towards the proteomes from Opisthokonta and plants, it also contains curated proteomes of species from other eukaryotic supergroups. We used combination of InterPro precomputed protein families as a final determiner for the affiliation to one of the PufSF members (IPR001313-Puf repeat, IPR040000Nop9, IPR040059-PUM3). In total, 7762 proteins were identified, and the proteins were classified according to hierarchic taxonomic groups (Fig. 2, Additional file 2: Table S2). Plotting the taxonomic distribution of the proteins showed that the highest number of PufSF proteins can be found in most of the plant species (Streptophyta), where the number of proteins ranged from 10 to 50. Extremely high number of proteins was also identified in ciliate Paramecium tetraurelia (43) but not in other ciliates or alveolates. In addition, all organism of analysed Euglenozoa group such as parasitic Trypanosoma and Leishmania species showed higher number of proteins (12-22). On the opposite end of the spectrum were parasites with reduced genomes, especially microsporidia or Cryptosporidium species with only one or two PufSF proteins. However, many animal taxa including insect and nematodes were also found to have only two or three proteins. Hence, at this point, we observed no clear relationship between the number of PufSF proteins and the biology or complexity of the surveyed organisms.
Three types of PufSF proteins have undergone different evolution in eukaryotes

Of all 7762 proteins from the reference proteomes, 1135 Nop9s, 5423 Pufs, and 1204 PUM3 proteins were identified (Additional file 2: Table S2). Interestingly, on average, each eukaryotic species contains a single Nop9 and PUM3 homologue and five Pufs (Fig. 3a). Moreover, while the number of Pufs is highly variable among lineages, the occurrence of single Nop9 and PUM3 proteins seems to be retained across eukaryotic diversity. The high number of PufSF proteins found in plants, Euglenozoa, and other species reflects lineage-specific amplification of the Puf proteins and not the ancestral state of early eukaryotes (Fig. 3a). The discrepancy observed between Nop9 and PUM3 gene copy number compared to Puf copy number might be related to different selective pressures experienced by these proteins owing to their role in the biogenesis of ribosomal RNA. On the other hand, given the Pufs' role in controlling the translation of multiple mRNAs-which will vary between organisms-it is possible that the number of Pufs will differ and could instead relate to the total number of the protein-coding genes in the cell. In order to test this hypothesis, we normalized the number of Pufs with respect to the total number of the protein-coding genes in the corresponding species (Fig. 3b) (Additional file 2: Table S2). Interestingly, the resulting ratio between Pufs and

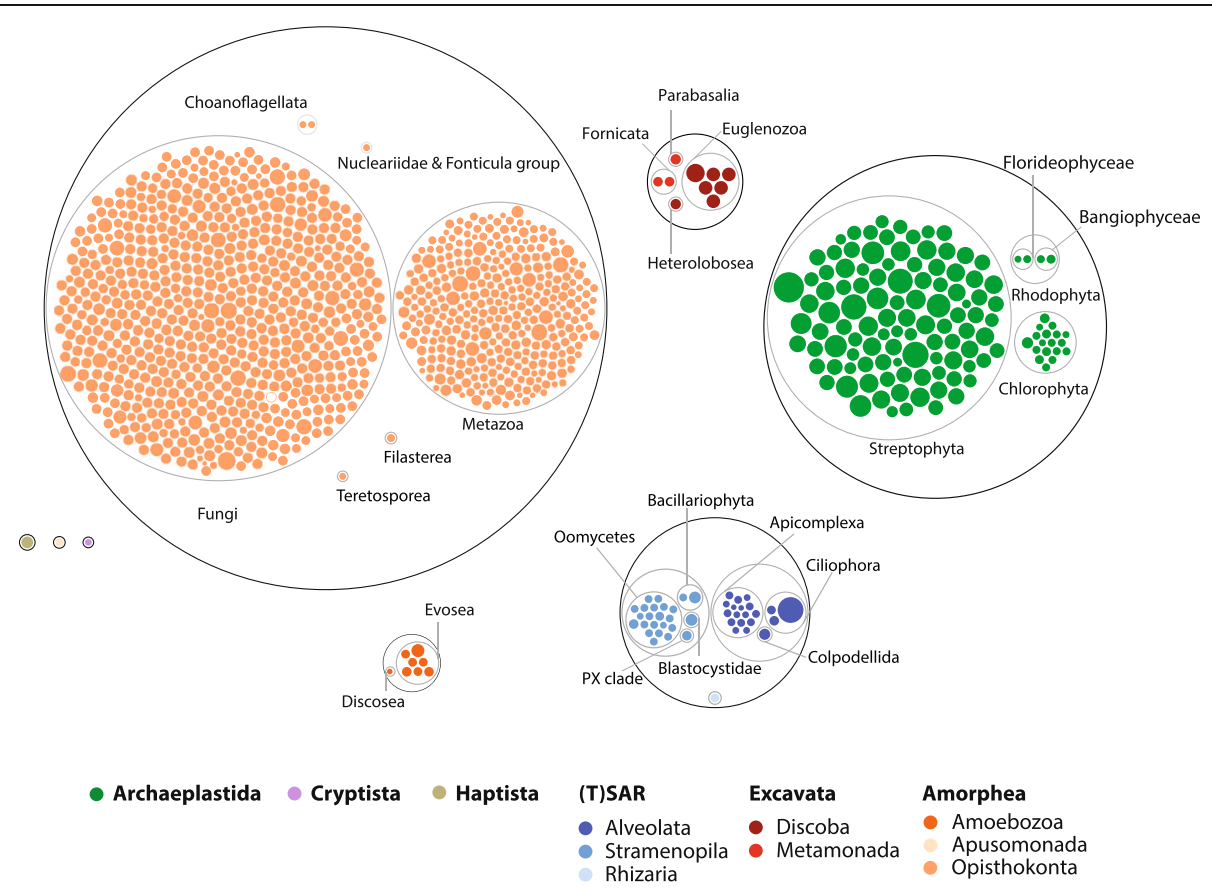

Fig. 2 PufSF proteins in major groups of eukaryotes. Puf family proteins identified in all UniProt reference proteomes were grouped according to taxonomic affiliation as specified in Additional file 2: Table S2 and showed in circle packing plot. Each dot corresponds to a genome of particular eukaryotic species, and the size of the dot represents a number of Puf family proteins in the predicted proteome and the colour-codes to the taxonomic affiliation to the eukaryotic supergroup. Grey circles depict taxonomic groups 

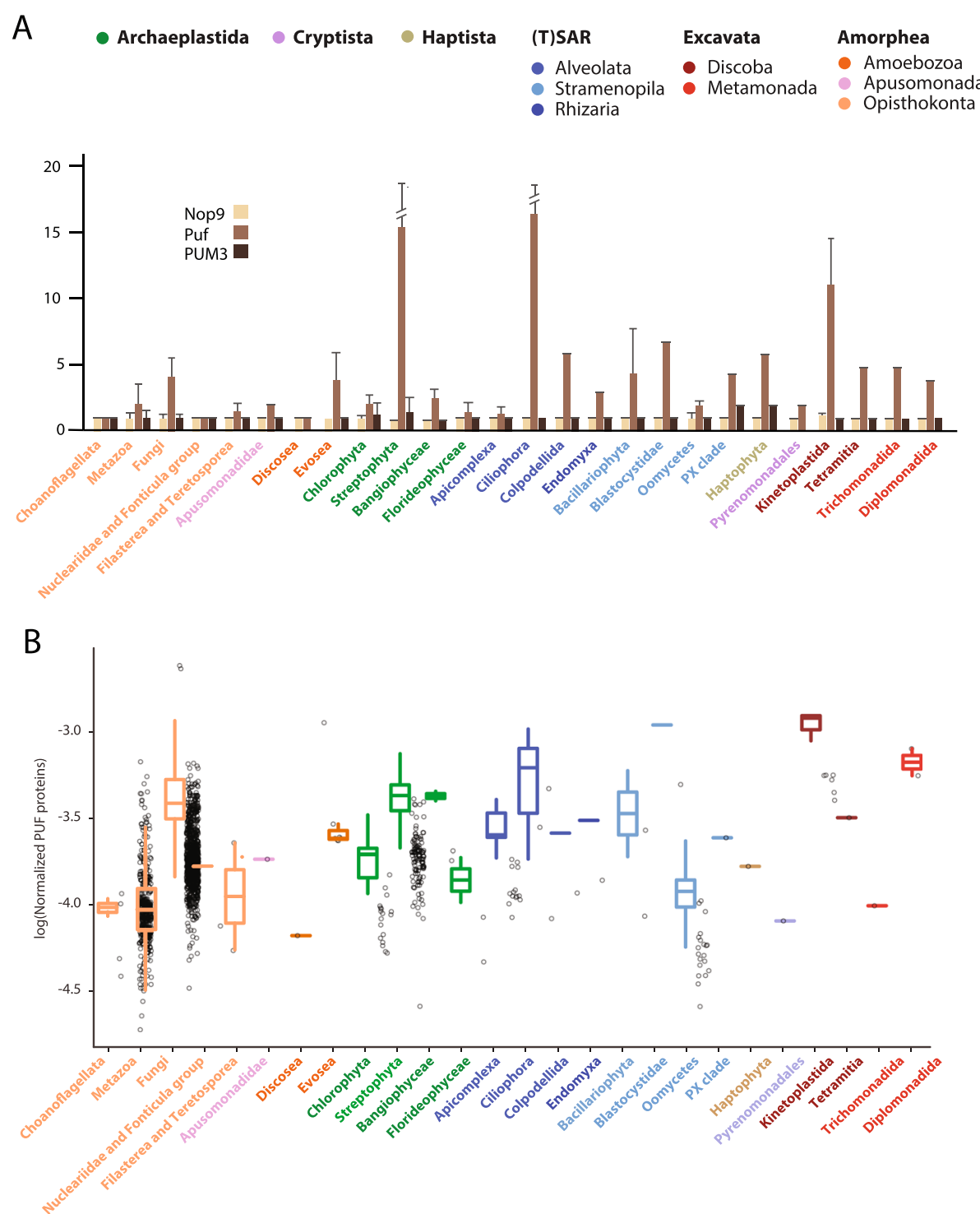

Fig. 3 Three types of PufSF proteins in major groups of eukaryotes. a The number of Nop9, Puf, and PUM3 orthologues was identified for each proteome in the dataset, and the values were averaged for the taxonomic group of eukaryotes. The error bars correspond to the standard deviation of variance of values within the particular taxonomic group. Error bars for Streptophyta and Ciliophora were cut for better visualization. b The number of Pufs normalized with respect to the total number of the protein coding; colour-codes correspond to the taxonomic affiliation to the eukaryotic supergroups

the pool of putative target transcripts seem to be very similar across eukaryotes (Fig. 3b) with the average number of one Puf for every $3.47 \times 10^{4}$ protein-coding genes.

\section{Phylogenetic reconstruction of PufSF proteins}

In order to get insight into the evolution of PufSF proteins, we performed the phylogenetic analyses on a dataset containing all three types of the proteins or the just a particular subset of either Puf, PUM3, or Nop9 orthologues (see the 'Materials and methods' section for more details). While the phylogenetic reconstructions proved to be problematic due to the repetitive structure of PufSF proteins, the overall tree (Fig. 4, Additional File 3:
Fig. S1) shows three distinct clades corresponding to Puf, PUM3, and Nop9. Subsequent subtrees of PUM3 and Nop9 proteins, which are present as single proteins, resolved all major eukaryotic groups with some unexpected position of orthologues mainly from the Metamonada supergroup (Additional File 3: Fig. S1), most likely caused by their high sequence divergence.

Given the presence of several Pufs in most eukaryotes, we endeavoured to resolve their evolutionary relationships and specifically if the presence of multiple Pufs reflects the ancestral state of LECA or rather they are independent paralogues arisen by linage-specific gene duplication(s). The phylogenetic reconstructions of Pufs 


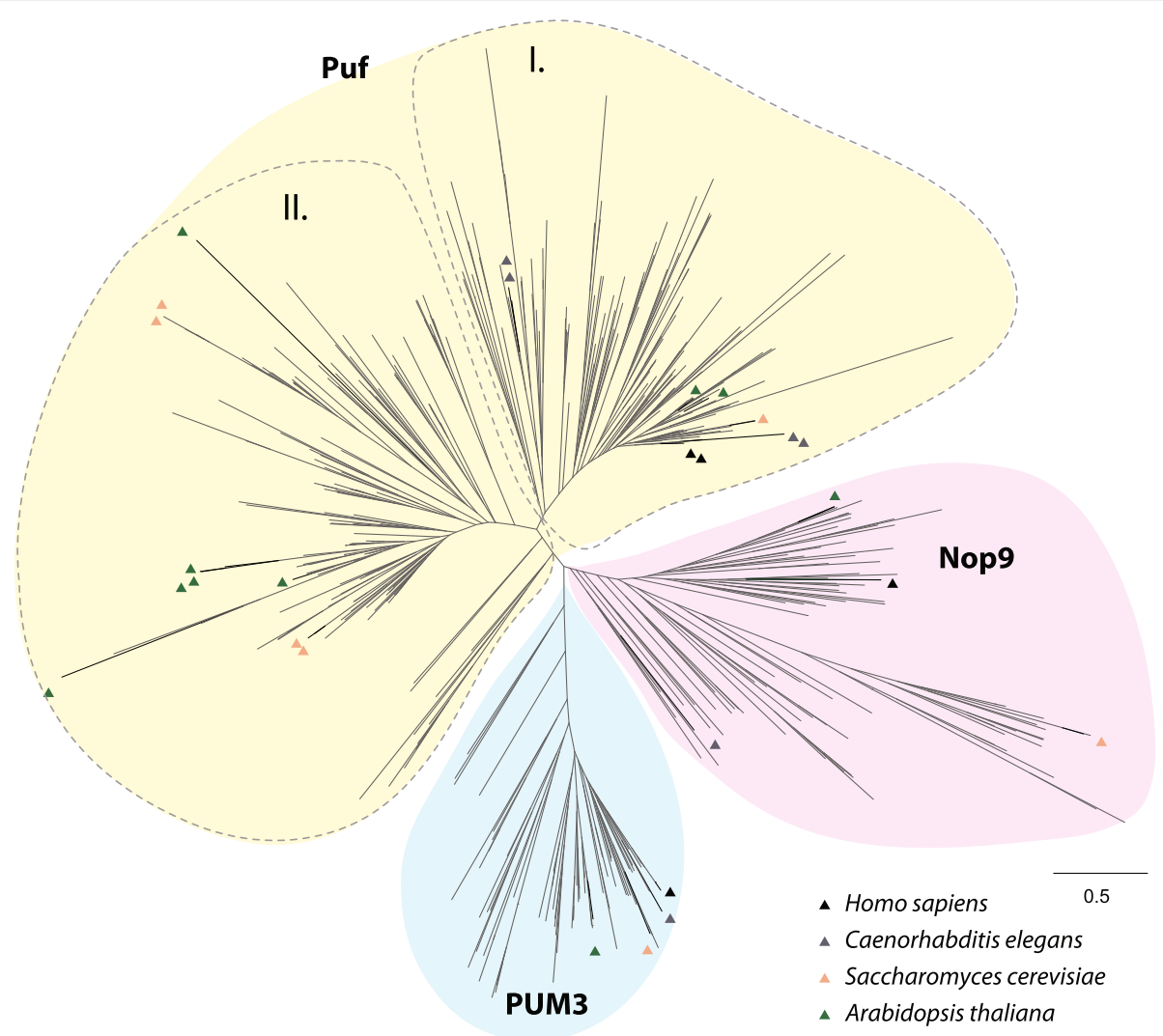

Fig. 4 Phylogenetic analysis of PufSF proteins. Maximum likelihood phylogenetic inference of PufSF proteins with sequences from $H$. sapiens, C. elegans, S. cerevisiae, and A. thaliana is shown as triangles with the indicated colours. For visualization purposes, support values were removed. Full phylogenies of the PUM3, Nop9, and Puf proteins can be found in Additional file 3: Fig. S1

remained very problematic, and despite using distinct alignment strategies, they did not return clear separation among individual Pufs and the eukaryotic taxa (Additional File 3: Fig. S1). However, we could identify two clear groups (labelled 'I' and 'II') (Fig. 4) that encompass the vast majority of eukaryotic taxa. This possibly reflects the presence of only two Pufs in LECA. Within group I and group II, there have been a number of lineage-specific duplications giving rise to the multitude of Pufs seen in different genomes.

\section{PufSF proteins in the metamonad G. intestinalis}

To test whether PufSF proteins are conserved in some of the most divergent eukaryotes, we specifically investigated the presence of PufSF proteins in G. intestinalis. Using a variety of sensitive sequence searching strategies (see the 'Materials and methods' section), we identified six proteins in G. intestinalis. The position and number of Puf repeats within the domain were predicted using HHpred and the alignments with the structurally characterized classical Pufs, Nop9, or PUM3 proteins, respectively. The classification of the proteins into the three types was confirmed by comparison with the domains defined at InterPro. Based on these classifications, we identified four $G$. intestinalis Puf homologues (GiPuf1-GiPuf4), one Nop9 (GiNop9), and PUM3 (GiPUM3) homologue (Fig. 5a). All four G. intestinalis Pufs were predicted to contain eight Puf repeats corresponding to eight TRMs (Fig. 5b), while both Nop9 and PUM3 homologues contained 11 Puf repeats similar to their homologues in Saccharomyces cerevisiae (Additional File 4: Fig. S2 and S3). The prediction of TRMs was performed by HHpred against the structurally characterized orthologues from S. cerevisiae and D. melanogaster (PDB ACNO. 5BZ1and 5KLA). However, the obtained TRMs for more divergent GiPuf1 and GiPuf2 were not in full agreement with the protein sequence alignment containing other G. intestinalis Puf proteins (Fig. 5b) as their Puf domain appeared shifted by two Puf repeats towards the C-terminus. At present, it is difficult to resolve if just the two Puf repeats were re-arranged or the entire domain was modified in these two proteins. 


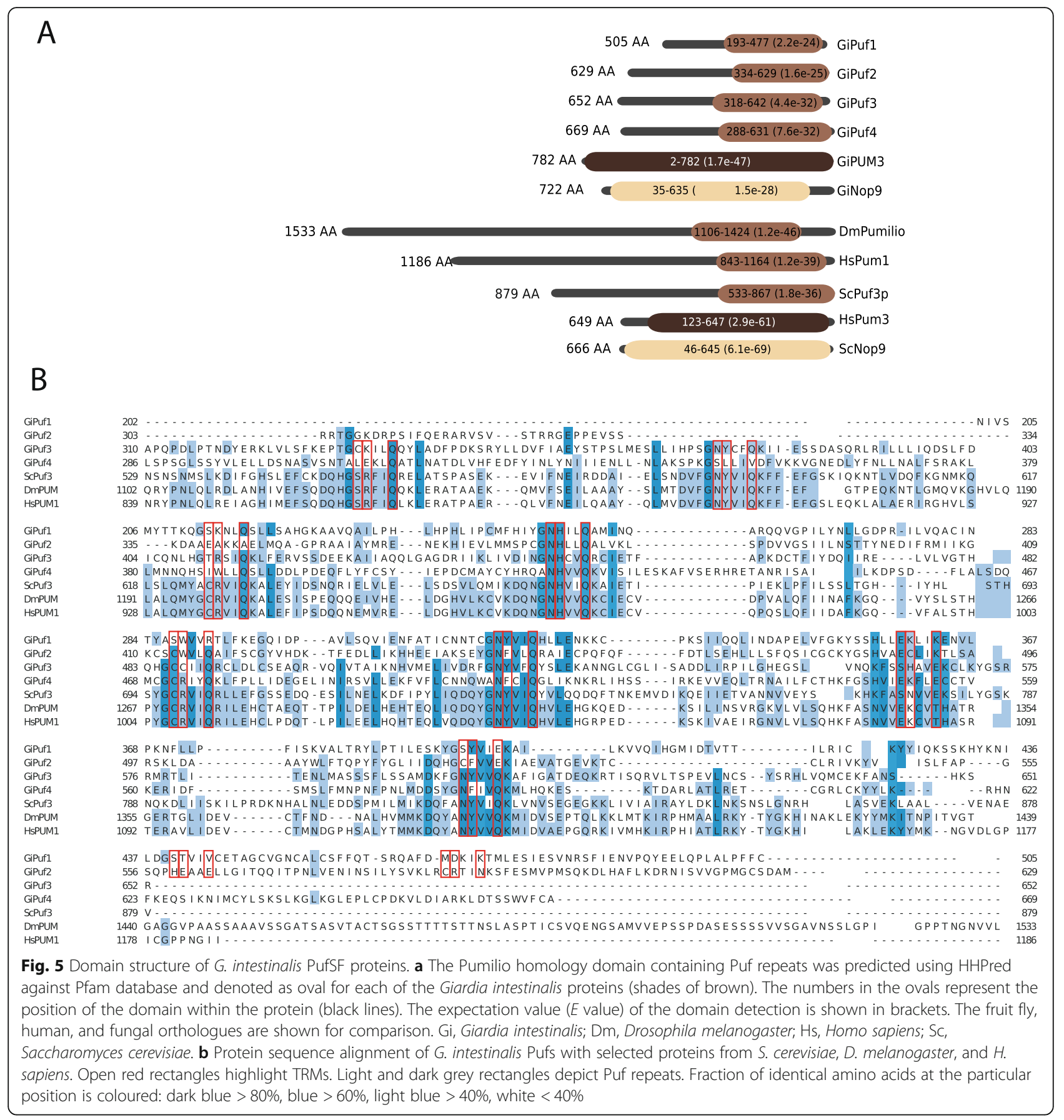

According to the phylogenetic reconstruction, the four G. intestinalis Pufs grouped together with the orthologues from closely related diplomonad species Spironucleus salmonicida and Trepomonas sp. distributed in group I and group II (Additional File 3: Fig. S1). GiPuf3, the most conserved Puf homologue of G. intestinalis, branched with group I Puf proteins while GiPuf1, GiPuf2, and GiPuf4 affiliated with the proteins from group II (Additional File 3: Fig. S1). The latter proteins thus likely represent lineage-specific gene duplications.

\section{Cellular localization of $G$. intestinalis PufSF proteins}

In general, PufSF proteins localize to the nucleus or cytosol. In the cytosol, Puf proteins often associate with the cytoplasmic face of cellular compartments [1].

To test the cellular localizations of each PufSF protein in G. intestinalis, we explored bioinformatic and experimental strategies. For bioinformatic predictions, we used DeepLoc [39], which uses neural networks to assess the localization of proteins based on a training set of experimentally localized proteins on UniProt. This algorithm 
predicted a cytoplasmic localization for all four G. intestinalis Pufs and Nop9 homologue and nuclear localization for only GiPUM3 (Fig. 6a). The cytosolic localization of the Pufs and the nuclear localization of GiPUM3 are in agreement with the expected roles of PufSF proteins, which control the stability and the localization of mRNAs in the cytosol and the nucleolar processing of 7S rRNA, respectively [2, 3]. However, given the role of Nop9 proteins in the maturation of pre-18S rRNA, the protein is expected to be in the nuclear compartment.

To test these subcellular localization predictions, we expressed all the G. intestinalis PufSF proteins with a Cterminal BAP (biotin acceptor peptide) tag in $G$.
A

Cyt Nuc CM ER Mit Extra $\begin{array}{lllllll}\text { GiPuf1 } & 0.915 & 0.081 & 0.001 & 0.000 & 0.000 & 0.000\end{array}$ $\begin{array}{llllllllllllll}\text { GiPuf2 } & 0.769 & 0.229 & 0.000 & 0.000 & 0.000 & 0.000\end{array}$ $\begin{array}{llllllllllllllll}\text { GiPuf3 } & 0.908 & 0.088 & 0.003 & 0.000 & 0.000 & 0.000\end{array}$ $\begin{array}{lllllllll}\text { GiPuf4 } & 0.882 & 0.114 & 0.002 & 0.000 & 0.000 & 0.000\end{array}$ $\begin{array}{llllllllll} & \text { GiPUM3 } & 0.233 & 0.761 & 0.002 & 0.001 & 0.001 & 0.001\end{array}$ $\begin{array}{lllllllllll}\text { GiNop9 } & 0.664 & 0.210 & 0.024 & 0.008 & 0.005 & 0.007\end{array}$

\section{C}

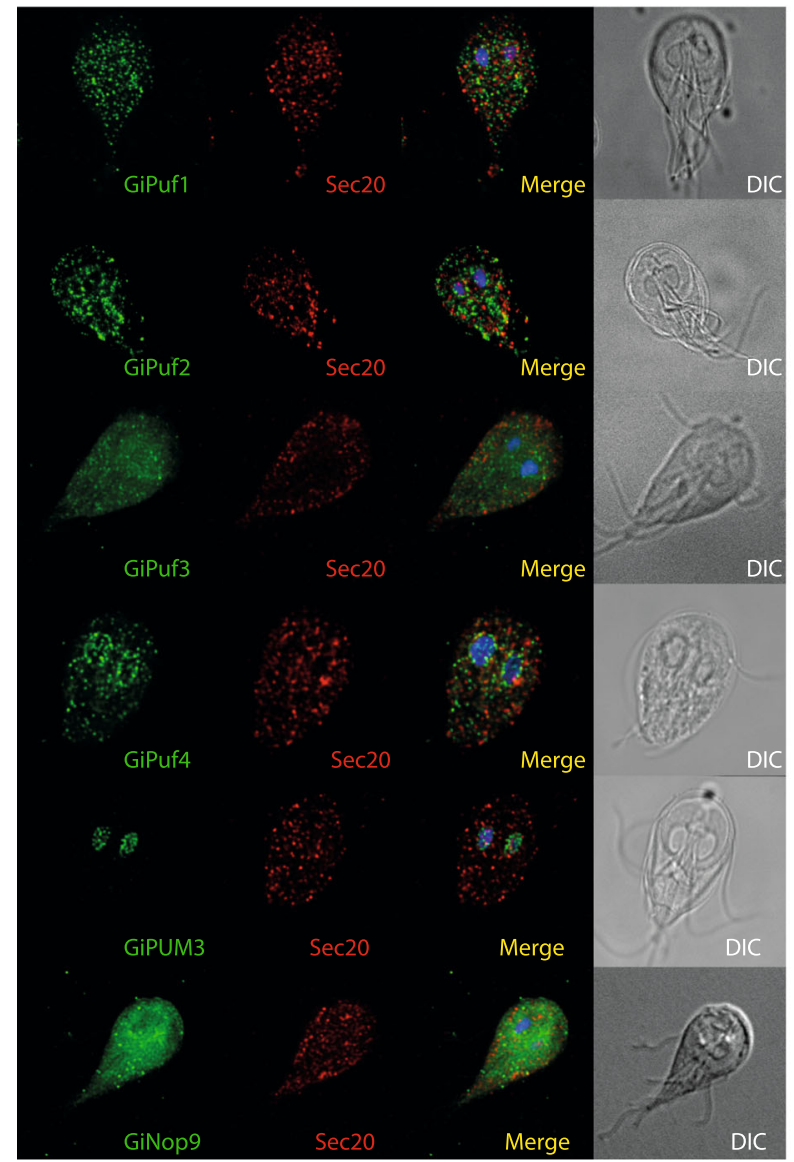

B

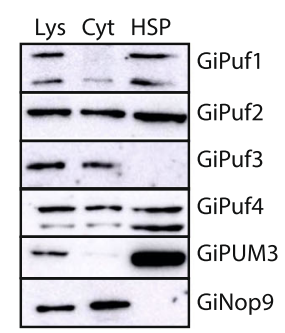

D
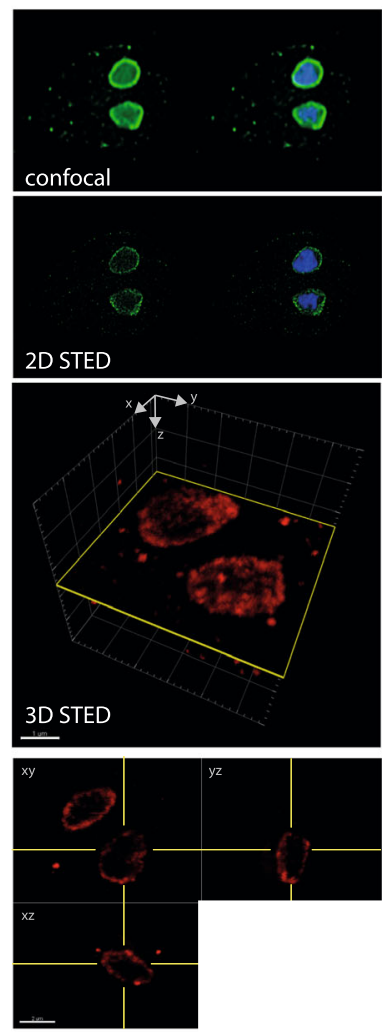

Fig. 6 Cellular localization of G. intestinalis Puf and Nop9 proteins. a The scores obtained by DeepLoc prediction indicate cytosolic localization of all but GiPUM3 protein, which is predicted as nuclear protein. Colouring gradient represents the values from 0 (red) to 1 (green). Cyt, cytosol; Nuc, nucleus; CM, cytoplasmic membrane; ER, endoplasmic reticulum; Mit, mitochondrion; Extra, extracellular. b Western blot analysis of G. intestinalis expressing BAP-tagged PufSF proteins (one of at least three independent cell experiments is show). SDS-PAGE and immunoblots show total lysate (Lys), cytosolic (Cyt), and high-speed pellet (HSP) fraction. c Immunofluorescence analysis of the same cell lines shows cellular localization of the proteins. PufSF proteins in green, Sec20-endomembrane system marker in red. DIC, Differential Interference Contrast. $\mathbf{d}$ Detailed imaging of GiPUM3 by confocal and 2D STED (GiPUM3 in green, DNA in blue). 3D STED of GiPUM3 with the orthogonal projections (GiPUM3 in red) 
intestinalis and analysed their localizations with cell fractionation and microscopy using antibodies directed at the BAP tag. Expression of all constructs but one (GiPuf4) was highly unstable and diminished quickly after establishing stable cell lines. Therefore, G. intestinalis cell lysates were obtained as soon as possible and separated into two fractions: (i) the high-speed pellet, containing sedimentable membrane-bound organelles such as the nucleus, endoplasmic reticulum, peripheral vacuoles, or mitosomes, and (ii) the cytoplasmic fraction [40] (Fig. 6b). In general, the Puf proteins showed three different types of distribution: GiPuf3 and GiNop9 were found specifically in the cytosolic fraction, while GiPuf1 and GiPUM3 were present predominantly in high-speed pellet fraction. Finally, GiPuf2 and GiPuf4 showed the presence in both cytosolic and high-speed pellet fractions indicating their partial association with the cellular membranes.

In agreement with the western blot analyses, the immunofluorescence confocal microscopy of GiPuf3 and GiNop9 showed mainly cytosolic localization of the proteins with some punctate distribution in the cell that do not co-localize with the endomembrane marker Sec20 (Fig. 6c). While the data are in agreement with the bioinformatic prediction, the cytosolic presence of G. intestinalis Nop9 homologue remains puzzling. GiPuf1, GiPuf2, and GiPuf4 were present in different kinds of vesicular structures likely corresponding to specific regions of the endomembrane system, which however did not co-localize, with our endomembrane marker protein Sec20 (Fig. 6c). In addition, GiPuf2 and GiPuf4 showed also a perinuclear staining, which indicated that the protein is associated with the nuclear membrane. Conversely, a very specific labelling of two G. intestinalis nuclei was observed for GiPUM3. In other eukaryotes, PUM3 localizes to discrete nucleolar spots in the nuclear matrix $[3,10]$. To determine the subnuclear localization of GiPUM3, we performed high-resolution STED microscopy. In both 2D and 3D STED microscopy, we observed GiPUM3 localizing to the periphery of the nucleus (Fig. 6d).

To determine potential Puf-interacting proteins in G. intestinalis, we explored the Puf-interactome using a high-resolution proximity labelling coupled to mass spectrometry. By determining potential interaction partners of the G. intestinalis Pufs, we could better predict their involvement in gene expression control. Unfortunately, the expression of tagged Pufs was highly unstable and diminished quickly after the cell transformation and we thus could not perform larger scale experiments required for protein- or RNA-pull down experiments. We were, however, able to generate a cell line weakly expressing BAP-tagged GiPuf4 in the presence of cytosolic biotin ligase BirA [41]. Upon crosslinking and purification of GiPuf4 on streptavidin-coupled Dynabeads, the triplicate samples were analysed by mass spectrometry. The purified GiPuf4 was found to be specifically enriched in our sample, although the experiment did not reveal any specific interacting partner protein above the statistical threshold (Additional file 5: Fig. S4, Additional file 6: Table S3). Thus, any functional predictions could not be drawn at this stage.

\section{Prediction of binding motifs of $G$. intestinalis Pufs and their target mRNA}

While we could not identify the interacting factors for G. intestinalis Pufs by mass spectrometry, we decided to predict the sets of recognized mRNAs for each homologue. The RNA sequence motif recognized by Puf/Nop9 proteins is determined by the combination of three amino acid residues, referred to as tripartite recognition motif (TRM). TRM is part of five residues in the second $\alpha$-helix of each Puf repeat represented as $1-2-\mathrm{X}$ $\mathrm{X}-5$ (where $\mathrm{X}$ is any hydrophobic residues). Within the TRM, positions 1 and 5 bind the edge of the RNA base, while the position 2 makes a stacking interaction with RNA molecule [42]. Some TRMs have been shown to be specific for particular base [11]. Hence, upon the identification of the TRMs in each Puf repeat, it is possible to predict its sequence-specific binding properties [9]. However, it should be noted that for some naturally occurring TRMs, the specificity has not been determined. By comparing the most closely related sequences to each G. intestinalis Puf identified with HHpred, we predicted the putative binding motif by manually checking the position of individual Puf repeats (Fig. 5b), and we could predict putative RNA-binding motifs for all G. intestinalis Pufs (Fig. 7a). Several predicted TRMs located in GiPuf1, GiPuf2, and GiPuf4 contained experimentally unidentified amino acid combinations which left these putative binding motifs incomplete. Interestingly, a complete binding motif predicted for GiPuf3 (5'UGUAUUUA-3') was found to be highly similar to $5^{\prime}$ UGUAUAUA-3'motif of prototypical members of the protein family such as human PUM1 or yeast Puf3 [43].

The predicted motifs of G. intestinalis Pufs were then used to search the dataset of theoretical 3 '-UTR of all 9747 G. intestinalis genes retrieved from GiardiaDB. Given that the 3'-UTRs of G. intestinalis mRNAs are very short [30, 32, 44], the length of the UTRs was limited to 50 bases only.

Using the FIMO (Find Individual Motif Occurrence) algorithm [45], a specific set of possible cognate mRNAs for GiPuf1-GiPuf4 was retrieved (Fig. 7, Additional file 8: Table S5). Each of the G. intestinalis Puf proteins was predicted to interact with the different number of transcripts, and this number was also inversely proportional to the G. intestinalis length of the predicted binding 
A

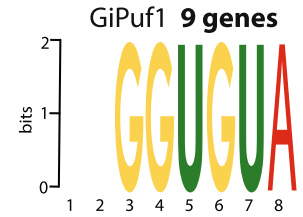

* GL50803_17350 Hypothetical protein GL50803_114442 Hypothetical protein GL50803_7272 GARP-like protein 1 GL50803 $6334 \quad$ Hypothetical protein GL50803 $95023 \quad$ Hypothetical protein GL50803-95023 Hypothein GL50803_86511 AcylCoAsynthetase GL50803_8460 Hypothetical protein GL50803_26228 Hypothetical protein GL50803_24537 Spindle pole protein

\section{GiPuf2 24 genes}

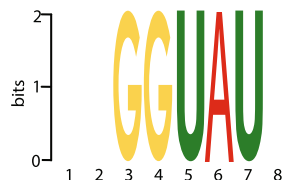

GL50803_106320 Zinc finger domain protein GL50803_16862 GL50803_33020 GL50803_9034 GL50803_113269 GL50803_15347 GL50803 7800 GL50803_14328 GL50803_11624 GL50803_103818 GL50803_117981 GL50803_6988 GL50803_4692 GL50803_10698 GL50803_10524 GL50803 6918 GL50803_7556 GL50803_36600 GL50803_8382 GL50803_15046 GL50803 7532 GL50803_95789 GL50803 14742 GL50803_10876 Kinase, NEK hypothetical protein hypothetical protein VSP

hypothetical protein hypothetical protein hypothetical protein hypothetical protein hypothetical protein hypothetical protein hypothetical protein hypothetical protein Phosphorylase kinase hypothetical protein DNA damage repair protein Lysosomal acid phosphatase hypothetical protein Short-chain dehydrogenase Ribosomal protein L26 Vacuolar ATP synthase Deoxyribonuclease Kinase, NEK hypothetical protein

\section{GiPuf3 7 genes}

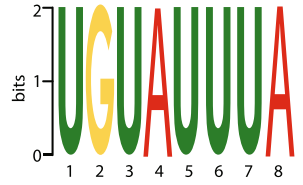

GL50803 115159 hypothetical protein GL50803_16454 Protein kinase, putative GL50803_97219 Fibrillarin-like pre-rRNA proc. $p$. GL50803_89285 hypothetical protein GL50803_89285 hypothetical protein GL50803_14487 hypothetical protein GL50803_23630 hypothetical protein GL50803_6188
GiPuf4 44 genes

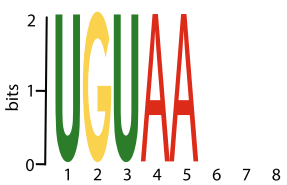

GL50803 6530

GL50803_16217

GL50803_6592

GL50803_36515

GL50803_19677

GL50803_99071

GL50803_41927

GL50803 4959

GL50803_3147

GL50803_14104

GL50803_7760

* GL50803_17350

GL50803_11621

GL50803_14375

GL50803_7018

GL50803_6408

GL50803_114307

GL50803_92246

GL50803_8974

GL50803_13851

GL50803_221691

GL50803_91643

GL50803_26603

GL50803_17143

GL50803 90434

GL50803_33301

GL50803_3414

GL50803_8496

GL50803_8917

GL50803_4587

GL50803_118786

GL50803 11521

GL50803_103916

GL50803_23833

GL50803_33232

GL50803_13438

GL50803_10231

GL50803_98892

GL50803 113723

GL50803_13075

GL50803_10773

GL50803 28888

GL50803 114636

GL50803_7026
Protein 21.1

NAG pyrophosphorylase hypothetical protein

hypothetical protein

hypothetical protein

hypothetical protein

hypothetical protein

Kinase, NEK

Protein 21.1

hypothetical protein

Sentrin

hypothetical protein

hypothetical protein

Asparaginyl-tRNA synthetase

hypothetical protein

SnRNP Sm-like protein, putative

Kinase, NEK

Cation-transporting ATPase 2

hypothetical protein

hypothetical protein

Histone methyltransferase

265 proteasome subunit

hypothetical protein

Pyruvate kinase

hypothetical protein

hypothetical protein

5-AMP-activated pr. kinase

Rac/Rho-like protein

Mu adaptin

hypothetical protein

VSP, putative

VSP

VSP, putative

Vacuolar protein sorting 35

GARP-like protein 4

Protein 21.1

TCP-1 chaperonin subunit zeta

hypothetical protein

hypothetical protein

hypothetical protein

hypothetical protein

hypothetical protein

hypothetical protein

hypothetical protein

B

Nop9 bound motif
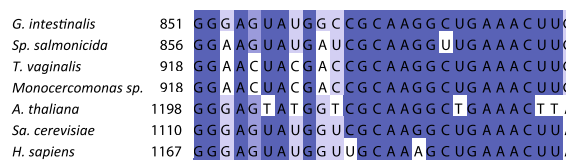

GAAGGCAUUGACGG AG

$G A A G G A \cup \cup G A C G G$
G AGAG
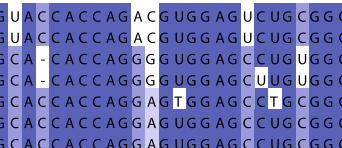

921

926

Fig. 7 (See legend on next page.) 
(See figure on previous page.)

Fig. 7 Predicted binding motifs of G.intestinalis PufSF proteins. a The tripartite recognition motifs (TRMs) of each Puf repeats of G.intestinalis Pufs were predicted, and the resulting sequence was used to search the conceptual transcriptome. The number of putative mRNA targets, which contain the motif in the $3^{\prime}-U T R$, is shown in bold. Asterisk denotes the same putative mRNA recognized by two different Pufs. b Sequence alignment of $18 \mathrm{~S}$ rRNA shows the conservation of the sequence recognized by Nop9 as it was experimentally identified for S. cerevisae Nop9. Fraction of identical nucleotides at the particular position is coloured: dark blue $>80 \%$, blue $>60 \%$, light blue $>40 \%$, white $<40 \%$

motif: where GiPuf3, GiPuf1, GiPuf2, and GiPuf4 were predicted to interact with $7,9,24$, and 44 transcripts, respectively. These numbers are substantially lower than other Puf proteins that are predicted to interact with hundreds of RNA targets $[19,46]$. We next explored the putative function and subcellular localization of the protein products of the predicted target transcripts (Additional file 8: Table S5). Interestingly, the target transcripts included other RNA-processing proteins (e.g. fibrillarin) and a component of the ERAD (endoplasmic reticulum-associated degradation) pathway (e.g. Derlin 1) (Additional file 8: Table S5).

Unlike Pufs, Nop9s use their 11 Puf repeats [21, 22] to specifically bind to the pre-18S rRNA at the central pseudoknot region [22] and regulate its processing possibly by competing with Nob1 nuclease [21]. However, how Nop9 TRMs interact with the rRNA was unknown until recently where it was shown that yeast Nop9 binds to a specific 11-nucleotide region of the pre-18S rRNA [22]. The alignment of $18 \mathrm{~S}$ rRNA sequences showed that this region is very well conserved across eukaryotes including species from the Metamonada group (Fig. 6b) suggesting that Nop9 might also recognize this region. However, in G. intestinalis, the cytoplasmic (and not nuclear) localization of this protein challenges whether GiNop9 and rRNA processing occurs in the nucleus.

Finally, when GiPUM3 was aligned with its characterized human and yeast counterparts, no previously identified TRMs could be identified within the sequence. This supported the absence of recognizable binding motif for this type of PufSF proteins (Additional File 4: Fig. S3) [2, 23].

\section{Discussion}

RBPs stand at the centre of the regulation of eukaryotic protein expression by mediating a wide range of posttranscriptional processes. There are several distinctive molecular properties of PufSF proteins $[4,5]$. The protein structure is composed of 8-11 units (Puf repeats), combination of which defines the sequence specificity towards their RNA targets. They have been shown to provide molecular basis for long sought processes such as site-specific translation of proteins, RNA degradation, or maturation of rRNA [1].

Here, we explored the evolution of PufSF proteins across the tree of eukaryotes. In addition to the originally characterized 'classical' Pufs $[4,5]$, which mediate the turnover of mRNAs via binding to their 3 '-UTRs
[19], recent studies in yeast model system have defined two additional types of PufSF proteins, which perform distinct cellular functions. Nop9 binds pre-18S rRNA during its maturation [3, 21, 22], and PUM3 orthologues are involved in processing 7S rRNA [2, 10, 47] and positioning of specific mRNAs [48].

Given that PufSF proteins are involved in the core processes of the eukaryotic cell (e.g. the maturation of rRNA and the translational control of mRNAs), we hypothesized that this system should be conserved across eukaryotic diversity. Indeed, we found that all majority lineages of eukaryotes encode at least one homologue of Nop9, PUM3, and two Puf proteins (Figs. 2, 3, and 4; Additional file 2: Table S2). The lack of PufSF proteins in prokaryotic genomes (including the closest prokaryotic relatives of eukaryotes-the Asgard archaea [49]) suggests that these proteins are in fact eukaryotic innovations. Moreover, their ubiquity across the tree of eukaryotes (including the highly diverged metamonads) suggests they were already present in protoeukaryotes before the emergence of LECA. To uncover the within protein family evolutionary history of the PufSF proteins, we performed a clustering analysis on the basis of sequence similarity. We found that PufSF proteins form three larger groups corresponding to the three independent biological functions performed by Puf, PUM3, and Nop9 proteins.

This suggests that the Puf and PUM3 proteins likely originated from the same ancestral gene. Interestingly, however, Nop9 and PUM3 proteins, despite belonging to separate groups of proteins within the family, share an array of 11 Puf repeats by which they interact with pre-18S [21] or 7S rRNA [2], respectively. On the basis of clustering analysis, it seems either that both Nop9 and PUM3 independently evolved into 11 Puf repeat containing proteins or that Pufs evolved from a larger 11-repeat domain ancestral protein into an 8-repeat domain protein by disposing of three Puf repeats. The latter seems to be more plausible as Pufs acquired additional long N-terminal part of mostly unknown function, which may, however, carry additional RNAbinding motifs [50].

As discussed above, it is most likely that the Nop9, PUM3, and two Puf proteins evolved during eukaryogenesis prior to the emergence of LECA, which was already indicated by the existence of four eukaryotic orthologous groups (KOGs) specific for PufSF [51]. The role of PufSF 
proteins in both nuclear and cytoplasmic processes suggests that this protein family likely coincided with the evolution of a nucleic acid compartment; however, the exact timing cannot be determined with current data. Indeed, as protoeukaryotes began to complexify, it is tempting to speculate that functional networks necessary to manage an expanding genome and gene repertoire would demand additional levels of regulation - a role potentially filled by RBPs such as the PufSF proteins. Future examination of the relative timing [52] of the emergence of PufSF proteins and their experimental characterization in diverse eukaryotic lineages will be critical in assessing these hypotheses.

The classification of the protein family members also revealed that while the number of Pufs is highly variable across lineages, both Nop9 and PUM3 orthologues remained present as single copy genes with no paralogous sequences in the majority of eukaryotes. This can likely be attributed to the conserved role of the Nop9 and PUM3 proteins in the maturation of rRNA which is essential for the biogenesis of eukaryotic ribosome. Our analysis also demonstrated that the expansion of Pufs in some lineages of eukaryotes is similar to those documented before for Arabidopsis thaliana [25]. Interestingly, we showed that the actual number of Pufs in eukaryotic supergroups corresponds to the size of the proteome and therefore also the number of putative target mRNAs within the cell. Importantly, our estimates are based on the total number of genes present in a genome and will therefore be an underestimation of the total number of transcripts present in a cell resulting in an overestimated Puf to total gene ratio depicted in Fig. 3. However, in organisms such as G. intestinalis or yeast, which are intron-poor and lack methods for generating splice variants, the total number of transcripts is approximately equal to the total number of proteincoding genes. Taking this into account, the Puf to total gene ratio of $G$. intestinalis is one of the highest across eukaryotic diversity. This suggests that while the total number of transcripts might still be determinative of Puf number in other organisms, additional factors beyond transcript number seem to govern the persistence of multiple Pufs in G. intestinalis.

The unique cellular and molecular features of G. intestinalis and its specialization make it an ideal model to study the extreme limits of cell biology [53]. With respect to RNA metabolism, G. intestinalis is often the exception to the rule. Previous studies have demonstrated that G. intestinalis lacks core regulatory components of transcription [37] and has even repurposed some conserved RNA metabolic pathways [54]. Our analyses of PufSF proteins from G. intestinalis have added at least one piece to the puzzle of RNA metabolism where we were able to show that like other eukaryotes, G. intestinalis has retained genes encoding all the three core RBPs.

However, the initial characterization of the proteins has uncovered interesting deviations from the eukaryotic blueprint. G. intestinalis PUM3 orthologue (GiPUM3) is localized on the nuclear periphery, which is different from nucleolar localization found in all experimentally analysed organisms $[10,48,55]$. Similarly, the cytosolic localization of G. intestinalis Nop9 orthologue contradicts its expected role in pre-18S rRNA processing occurring in the nucleolus.

This raises a question on the nature and localization of G. intestinalis nucleolus. For long time, G. intestinalis has been thought to lack nucleolus-like structures since nucleolar markers have failed to show discrete nuclear labelling [56]. However, later hybridization of $18 \mathrm{~S}$ rRNA and localization of fibrillarin, pseudouridine synthase, and snoRNA suggested specific distribution of the nucleoli on the periphery of both G. intestinalis nuclei [5760]. It is therefore likely that the nuclear periphery functionally replaces nucleolar bodies typical of other eukaryotes [31].

Unexpectedly, we observed that GiNop9 localizes to the cytosol of G. intestinalis. We have a number of hypotheses that could explain this observation. First, we cannot rule out that the overexpression of the protein resulted in mistargeting of G. intestinalis Nop9, although we should be able to observe at least partial nuclear signal. Alternatively, GiNop9 does not participate in pre$18 \mathrm{~S}$ rRNA processing in G. intestinalis or its cytosolic presence is a consequence of unique nucleolar localization in G. intestinalis. Nop9 is thought to prevent rRNA processing by cytosolic Nob1 nuclease by competing for binding at the same rRNA sequence [21]. Nob1 mediates the late step of pre-rRNA processing which generates 18S rRNA from 20S intermediate rRNA [61], and these interactions thus occur at the interface between the nucleus and cytosol. It is possible that in $G$. intestinalis, the relocation of the nucleolus to the nuclear periphery also relocated the latter steps of rRNA processing outside the nucleus whereby GiNop9 participates in the cytosolic maturation of the ribosome.

The bioinformatic survey for putative target mRNAs, whose 3 '-UTR are bound by one of four G. intestinalis Pufs, returned only a small set of candidate genes compared to other eukaryotes [8]. This might stem from the extremely short UTRs in G. intestinalis [32, 37], which limits the actual region of interaction with RBPs. We could only confidently predict a complete binding motif (5' - UGUAUUUA-3') for GiPuf3, and in this case, the motif was found to be highly similar to binding motifs of Pufs from other lineages of eukaryotes. The conservation of this binding motif in G. intestinalis was surprising given that G. intestinalis usually lacks many other core 
regulatory elements when compared to model eukaryotes (e.g. TATA box or Inr element) [62, 63]. Given the theoretical capabilities of Pufs to bind any possible RNA sequence [64], it raises an important question on the functional advantage of this particular binding motif in RNA-protein interactions.

Obviously, there are many unknowns left concerning the function of PufSF proteins especially in non-model organisms like G. intestinalis and other protists. However, despite numerous differences in posttransriptional processes found among eukaryotes, this work shows that PufSF proteins have constituted key RNA-processing proteins since LECA.

\section{Conclusions}

In this study, we show that all three types of PufSF proteins are found across eukaryotic diversity. Moreover, we found that their role in both rRNA maturation (Nop9, PUM3) and mRNA translational control (Pufs) is conserved in all major lineages of eukaryotes and that they were present in the last eukaryotic common ancestor (LECA). Finally, we experimentally show the presence of three types of PufSF family proteins in a Metamonad G. intestinalis and highlight their several intriguing lineagespecific adaptations.

\section{Materials and methods}

Bioinformatic analyses

For the clustering analyses, the entry dataset of PufSF proteins was obtained from InterPro/UniProt database. All duplicates and sequences shorter than 500 amino acids were filtered out. Clustering analysis with CLANS was performed using MPI Bioinformatics toolkit available at https://toolkit.tuebingen.mpg.de/ [38]. The resulting 2D coordinates were plotted in GraphPad, and the dots representing the protein sequences were colour-coded according to current taxonomic descriptors of eukaryotes [65]. To identify putative Puf homologues in G. intestinalis, the protein alignment of PufSF was used in HHpred search (available at https://toolkit. tuebingen.mpg.de/\#/tools/hhpred) against conceptual G. intestinalis proteome (Giardia_lamblia_ATCC_50803_ 31_Aug_2017) [66]. For the detection of Puf-binding motifs in 3'-UTRs, the motifs were generated by MEME (Multiple Em for Motif Elicitation available at http:// meme-suite.org/tools/meme) [67] and used by FIMO (Find Individual Motif Occurrences) program (available at http://meme-suite.org/tools/fimo) [45] against our custom database of all conceptual 50-nt-long 3'-UTRs (obtained from GiardiaDB http://giardiadb.org/giardiadb/) [68]. Dataset of $18 \mathrm{~S}$ rRNA was obtained from the high-quality ribosomal RNA database SILVA (available at https://www.arb-silva.de/) [69]. Sequences were aligned using mafft -auto [70], and alignment was coloured according to percentage identity in Jalview [71]. The proteins co-purified with GiPuf4 and identified by mass spectrometry were analysed by HHpred for homology detection and by DeepLoc (available at http:// www.cbs.dtu.dk/services/DeepLoc/) for subcellular localization [39].

\section{Classification of PufSF proteins in eukaryotes}

To assess the distribution of Nop9, PUM3, and Puf sequences across the tree of eukaryotes, we used the precomputed domain annotation on InterProScan (ftp://ftp. ebi.ac.uk/pub/databases/interpro/protein2ipr.dat.gz) of all eukaryotes present in the Reference Proteome database from UniProtKB. Importantly, since we were interested in understanding how many gene expansions occurred in each genome, we ignored splice variants by selecting only one representative protein per variant. Proteins were classified based on the presence of the following IPR domains/PANTHER family/SMART domain: Nop9 (IPR040000/PTHR13102), PUM3 (IPR040059/ PTHR13389), and Puf (IPR001313/SM00025).

\section{Phylogenetic dataset construction and analyses}

For phylogenetic dataset construction, a subset of representative taxa across eukaryotic diversity were selected from the entry dataset from the clustering analysis. Due to the variety of different protein lengths, only the region corresponding to the Puf repeats was analysed; for each sequences, we extracted the region corresponding to the start position of the first, until the last position of the final, Puf repeat using HMMsearch with the SMRT profile (SM00025). Sequences were aligned using mafft-linsi [70], and ambiguously aligned sites were removed using trimal with the '-gappyout' option [72]. Initial phylogenetic tree of the entire PufSF (i.e. Puf, Nop9, and PUM3 proteins) was generated using FastTree with the -lg option [73]. This tree was manually examined, and in the cases where an organism had multiple copies of a paralogue that were monophyletic, only one sequence was retained. This refined dataset was realigned as above. Maximum likelihood inference of the reduced dataset was performed using IQ-TREE v 2.0 [74] using the LG + $\mathrm{G}+\mathrm{F}$ model. Given the large size of the dataset, we did not perform model testing with the mixture models for the PufSF tree. A total of $1000 \mathrm{SH}$-aLRTs (SH-like approximate likelihood ratio test; -alrt 1000), 1000 ultrafast bootstraps (-bb 1000) [75], 100 non-parametric boostraps (-b 100), and the transfer bootstrap expectation calculation (--tbe) [76] were performed and mapped onto the best-scoring maximum likelihood tree. To refine the phylogeny of Nop9, PUM3, and Puf proteins, we analysed each subclade separately. The sequences were aligned as above. Maximum likelihood inference of the reduced dataset was performed using IQ-TREE v 2.0 
[74] under the best-scoring model of evolution selected by ModelFinder supplemented with the $\mathrm{C}$-series mixture models using the -mset option [77]. A total of $1000 \mathrm{SH}-$ aLRTs (SH-like approximate likelihood ratio test; -alrt 1000), 1000 ultrafast bootstraps (-bb 1000) [75], were mapped onto the best-scoring maximum likelihood tree. The resulting maximum likelihood tree was used as the guide tree $(-\mathrm{ft})$ for rapid approximation of posterior mean site frequency (PMSF) of the C-series of mixture models [78] to generate 100 non-parametric bootstraps (-b 100) together with the transfer bootstrap expectation (--tbe). Alignment features and model parameters are specified in Additional file 7: Table S4, and datasets are available on the figshare repository (DOI:https://doi.org/ 10.6084/m9.figshare.12097692), see 'Availability of Data and Materials'.

\section{Cell culture, cloning, and transfection}

The G. intestinalis strain WB (ATCC 30957) was grown in TYI-S-33 medium [79] supplemented with $10 \%$ heatinactivated bovine serum, $0.1 \%$ bovine bile, and antibiotics at $37{ }^{\circ} \mathrm{C}$. The genes encoding G. intestinalis PufSF proteins [GiardiaDB accession numbers GL50803_17262 (GiPuf1), GL50803_17590 (GiPuf2), GL50803_17325 (GiPuf3), GL50803_4548 (GiPuf4), GL50803_16602 (GiPUM3), and GL50803_14117 (GiNop9)] and 150-bplong 5'-UTR of GiPuf1, GiPuf2, GiPuf3, GiPuf4, and GiNop9 were amplified from genomic DNA using PCR and inserted to the plasmid pOndra [40] with the Cterminal biotin acceptor peptide (BAP). Additional file 9: Table S6 in lists all primers used in this study. Escherichia coli biotin ligase (BirA) in pTG vector was used for the co-expression with GiPuf4 [80]. G. intestinalis transfection was performed as previously described [40]. Briefly, $1 \times 10^{7}$ cells were electroporated with a Bio-Rad Gene Pulser using an exponential protocol $(U=350 \mathrm{~V}$; $C=1,000 \mu \mathrm{F} ; R=750 \Omega$ ). The transfected cells were grown in medium supplemented with antibiotics $(57 \mu \mathrm{g} /$ $\mathrm{ml}$ puromycin and $600 \mu \mathrm{g} / \mathrm{ml} \mathrm{G418).}$

\section{Cell fractionation}

Trophozoites of G. intestinalis in logarithmic growth were harvested in ice-cold phosphate-buffered saline (PBS, pH 7.4) at $1000 \times g$ at $4{ }^{\circ} \mathrm{C}$ for $10 \mathrm{~min}$, washed in SM buffer (20 mM MOPS, $250 \mathrm{mM}$ sucrose, pH 7.4), and collected by centrifugation at $1000 \times g$, for $10 \mathrm{~min}$ at $4{ }^{\circ} \mathrm{C}$. The pellet of the cells was resuspended in SM buffer supplemented with protease inhibitors (Roche). The cells were lysed on ice by sonication with 1-s pulses and $40 \%$ amplitude for $2 \mathrm{~min}$ (Sonicator ultrasonic processor Q125, Qsonica). The lysate was subjected to centrifugation at $2680 \times g$, for $20 \mathrm{~min}$ at $4{ }^{\circ} \mathrm{C}$ to sediment nuclei, cytoskeleton, and remaining unbroken cells. The supernatant was subjected to centrifugation at $180,000 \times g$, for
$30 \mathrm{~min}$ at $4{ }^{\circ} \mathrm{C}$. The resulting supernatant corresponded to cytosolic fraction, and the high-speed pellet (HSP) contained organelles including the mitosomes and the endoplasmic reticulum.

\section{Crosslinking, protein isolation, and mass spectrometry (MS)}

G. intestinalis cells were grown in TYI-S-33 medium enriched with $50 \mu \mathrm{M}$ biotin for $24 \mathrm{~h}$ before harvesting. The cells were lysed by sonication, and the cell lysate ( 25 $\mathrm{mg}$ of total protein) was used for the protein isolation. The sample was diluted to final protein concentration of $3 \mathrm{mg} / \mathrm{ml}$ in PBS (pH 7.4) and supplemented with protease inhibitors (Roche). Crosslinker DSP (dithiobis [succinimidyl propionate]; Thermo Scientific) was added to the final concentration of $200 \mu \mathrm{M}$, and the sample was incubated 1 $\mathrm{h}$ on ice. The reaction was stopped by adding $50 \mathrm{mM}$ final concentration of Tris $(\mathrm{pH} \mathrm{8.0)}$ for $15 \mathrm{~min}$ at room temperature. The sample was diluted 5 times with the boiling buffer (50 mM Tris, $1 \mathrm{mM}$ EDTA, 1\% SDS, pH 7.4), incubated at $80^{\circ} \mathrm{C}$ for $10 \mathrm{~min}$, and collected by centrifugation at $30,000 \times g$, for $10 \mathrm{~min}$ at room temperature. Supernatant was diluted 1:10 in the binding buffer (50 $\mathrm{mM}$ Tris, $150 \mathrm{mM} \mathrm{NaCl}, 5 \mathrm{mM}$ EDTA, $1 \%$ Triton X-100, $\mathrm{pH}$ 7.4). Meanwhile, $100 \mu \mathrm{l}$ of streptavidin-coupled magnetic beads (Dynabeads MyOne Streptavidin C1, Invitrogen) was washed 3 times in the binding buffer using a magnetic stand (according to the instructions of the manufacturer). The beads were mixed with the supernatant, and the sample was incubated for $16 \mathrm{~h}$ at $4{ }^{\circ} \mathrm{C}$ with gentle rotation. The magnetic beads were washed 3 times in the binding buffer with $0.1 \%$ sodium deoxycholate (SDC) for $5 \mathrm{~min}$, once in boiling buffer for $5 \mathrm{~min}$, once in washing buffer ( $60 \mathrm{mM}$ Tris, 2\% SDC, 10\% glycerol) for 5 $\mathrm{min}$, and finally twice in $100 \mathrm{mM}$ triethylammonium bicarbonate (TEAB) with $0.1 \%$ SDC for $5 \mathrm{~min}$. One tenth of the beads was used to analyse the efficiency of the procedure. Specifically, the sample was mixed with the SDSPAGE sample buffer containing $20 \mathrm{mM}$ biotin and incubated at $95^{\circ} \mathrm{C}$ for $5 \mathrm{~min}$ to elute the proteins from the beads. The samples were resolved on 12\% SDS-PAGE and transferred to nitrocellulose membrane. For immunodetection, the following antibodies were used: $1^{\circ}$ anti-BAP (1:1000) (Genscript) and $2^{\circ}$ anti-rabbit polyclonal antibody coupled to HRP (1:2000) (Sigma). Remaining beads with bound proteins were submitted to mass spectrometry analysis.

\section{Tandem mass spectrometry (MS/MS) analyses}

The beads were incubated at $5 \mathrm{mM}$ tris (2-carboxyethyl) phosphine (TCEP) at $60^{\circ} \mathrm{C}$ for $1 \mathrm{~h}$ to reduce the disulfide bridges and blocked with $10 \mathrm{mM}$ final concentration of methyl methanethiosulfonate (MMTS) for $10 \mathrm{~min}$ at $37^{\circ} \mathrm{C}$. Samples were cleaved on the beads with $1 \mu \mathrm{g}$ of 
trypsin at $37^{\circ} \mathrm{C}$ for $16 \mathrm{~h}$. After digestion, samples were centrifuged, and supernatants were collected and acidified with trifluoroacetic acid (TFA at a final concentration of 1\%). SDC was removed by extraction with ethylacetate [81]. Peptides were desalted on Michrom C18 column. Nano Reversed phase column (EASY-Spray column, $50 \mathrm{~cm} \times 75 \mu \mathrm{m}$ ID, PepMap C18, $2 \mu \mathrm{m}$ particles, $100 \AA$ pore size) was used for LC/MS analysis. Mobile phase buffer $\mathrm{A}$ was composed of water and $0.1 \%$ formic acid. Mobile phase B was composed of $0.1 \%$ formic acid in acetonitrile. Samples were loaded onto the trap column (Acclaim PepMap300, C18, $5 \mu \mathrm{m}, 300 \AA$ Wide Pore, $300 \mu \mathrm{m} \times 5 \mathrm{~mm}, 5$ Cartridges) for $4 \mathrm{~min}$ at $15 \mu \mathrm{l} / \mathrm{min}$. Loading buffer was composed of water, $2 \%$ acetonitrile, and $0.1 \%$ TFA. Peptides were eluted with mobile phase $\mathrm{B}$ gradient from 4 to $35 \% \mathrm{~B}$ in $1 \mathrm{~h}$. Eluting peptide cations were converted to gas-phase ions by electrospray ionization and analysed on a Thermo Orbitrap Fusion (Q-OT-qIT, Thermo). Survey scans of peptide precursors from 400 to $1600 \mathrm{~m} / \mathrm{z}$ were performed at $120 \mathrm{~K}$ resolution (at $200 \mathrm{~m} / \mathrm{z}$ ) with a $5 \times 10^{5}$ ion count target. Tandem MS was performed by isolation at 1.5 Th with the quadrupole, HCD fragmentation with normalized collision energy of 30, and rapid scan MS analysis in the ion trap. The MS 2 ion count target was set to 104, and the max injection time was $35 \mathrm{~ms}$. Only those precursors with charge state 2-6 were sampled for MS 2 . The dynamic exclusion duration was set to $45 \mathrm{~s}$ with a $10-\mathrm{ppm}$ tolerance around the selected precursor and its isotopes. Monoisotopic precursor selection was turned on. The instrument was run in top speed mode with 2 -s cycles [82]. All data were analysed and quantified with the MaxQuant software (version 1.5.3.8) [83]. The false discovery rate (FDR) was set to $1 \%$ for both proteins and peptides, and we specified a minimum length of seven amino acids. The Andromeda search engine was used for the MS/MS spectra search against the G. intestinalis database (downloaded from UniProtKB in September 2017, containing 12,665 entries). Enzyme specificity was set as C-terminal to Arg and Lys, also allowing cleavage at proline bonds and a maximum of two missed cleavages. Dithiomethylation of cysteine was selected as fixed modification and $\mathrm{N}$ terminal protein acetylation and methionine oxidation as variable modifications. The 'match between runs' feature of MaxQuant was used to transfer identifications to other LC-MS/MS runs based on their masses and retention time (maximum deviation $0.7 \mathrm{~min}$ ), and this was also used in quantification experiments. Quantifications were performed with the label-free algorithms described recently [83]. Data analysis was performed using Perseus 1.5.2.4 software [84]. The mass spectrometry proteomics data have been deposited in the ProteomeXchange Consortium via the
PRIDE [85] partner repository with the dataset identifier PXD019608.

\section{Immunofluorescence microscopy}

G. intestinalis trophozoites were fixed in $1 \%$ paraformaldehyde for $30 \mathrm{~min}$ at $37^{\circ} \mathrm{C}$ and collected by centrifugation at $1000 \times g$ for $5 \mathrm{~min}$ at room temperature. The cells were washed in PEM buffer (100 mM PIPES pH 6.9, 1 $\mathrm{mM}$ EGTA, and $0.1 \mathrm{mM} \mathrm{MgSO}_{4}$ ) and placed on cover slips. The cells were permeabilized by $0.2 \%$ Triton X-100 (Sigma-Aldrich) for $20 \mathrm{~min}$, washed three times with $1 \times$ PEM buffer, and incubated with primary antibodies in PEMBALG (100 mM PIPES pH 6.9, 1 mM EGTA, 0.1 $\mathrm{mM} \mathrm{MgSO}_{4}, 1 \% \mathrm{BSA}, 0.1 \% \mathrm{NaN}_{3}, 100 \mathrm{mM}$ lysine, and $0.5 \%$ cold-water fish skin gelatin) for $1 \mathrm{~h}$. Cells were probed with the following primary antibodies: rat antiHA monoclonal IgG antibody (1:1000 dilution), mouse anti-BAP monoclonal antibody (1:1000 dilution), and rabbit anti-Sec20 polyclonal [86] (1:1000 dilution). The cover slips were washed three times with $1 \mathrm{ml}$ of $1 \times$ PEM and were incubated with secondary antibodies Alexa Fluor 488-conjugated goat anti-rat IgG and Alexa 594-conjugated donkey anti-rabbit IgG or Alexa594conjugated goat anti-mouse IgG for $1 \mathrm{~h}$. After $3 \times 5 \mathrm{~min}$ washes in PEM buffer, slides were mounted with Vectashield containing DAPI (4', 6-diamidino-2-phenylindole; Vector Laboratories) or Hoechst solution (33258). Stimulated emission depletion (STED) microscopy was performed on a commercial Abberior STED 775 QUAD scanning microscope (Abberior Instruments $\mathrm{GmbH}$, Germany) equipped with Ti-E Nikon body, QUAD beam scanner, Easy3D STED Optics Module, and Nikon CFI Plan Apo Lambda objective (× 60 Oil, NA 1.40). Samples were illuminated by pulsed $561-\mathrm{nm}$ and $640-\mathrm{nm}$ lasers and depleted by a pulsed 775-nm STED laser of 2D donut shape (all lasers: $40 \mathrm{MHz}$ repetition rate). Fluorescence signal was detected with single photon counting modules (Excelitas Technologies). Line-interleaved acquisition enabled separated detection of individual channels in spectral range from 605 to $625 \mathrm{~nm}$ and from 650 to $720 \mathrm{~nm}$. The confocal pinhole was set to $1 \mathrm{AU}$.

\section{Supplementary information}

Supplementary information accompanies this paper at https://doi.org/10. 1186/s12915-020-00814-3.

Additional file 1: Table S1. The clustering analysis of PufSF proteins. Additional file 2: Table S2. Classification of PufSF proteins from UniProt referenced proteomes.

Additional file 3: Figure S1. Phylogenetic analysis NOP9, PUM3 and PUF proteins. Full phylogenies of maximum-likelihood analysis of PUM3, Nop9 and Puf proteins with SH-like approximate likelihood ratio test, ultrafast bootstrap supports, and with both non-parametric Felsenstein's Bootstrap Proportion (FBP) supports (i.e., PMSF) and Transfer Bootstrap 
Expectation (TBE) supports as indicated. See Additional file 7 for alignment properties and model parameters.

Additional file 4: Figure S2. Proteins sequence alignment of Nop9 orthologues. Figure S3. Protein sequence alignment of PUM3 orthologues.

Additional file 5: Figure S4. Volcano plot of proteomics analysis of GiPuf4 pull down.

Additional file 6: Table S3. Proteomics analysis of GiPuf4 pull down.

Additional file 7: Table S4. Alignment properties and model parameters used for the phylogenetic analyses.

Additional file 8: Table S5. Prediction of Giardia Pufs target mRNA. Additional file 9: Table S6. Primers used in the study.

\section{Acknowledgements}

We would like to thank Nikola Polanska for the help with the bioinformatic analyses.

\section{Authors' contributions}

$P D, V N$, and $C S$ designed the experiments; $V N, C S, L V$, and MV performed the experiments; VN, CS, PD, LV, and MV analysed the data; NV, CS, and PD wrote the manuscript. All authors read and approved the final manuscript.

\section{Funding}

The project was supported by the GAUK grant 1071016 and PRIMUS grant PRIMUS/SCV34 from Charles University; by the Czech Science Foundation grant 20-25417S; by the National Sustainability Program II (Project BIOCEVFAR, LQ1604) from the Ministry of Education, Youth and Sports of CR (MEYS); and by the project 'Centre for research of pathogenicity and virulence of parasites' (no. CZ.02.1.01/0.0/0.0/16_019/0000759) funded by the European Regional Development Fund. Microscopy was performed in the ICF co-financed by the European Regional Development Fund and the state budget of the Czech Republic, project nos. CZ.1.05/4.1.00/16.0347 and CZ.2.16/3.1.00/21515.

\section{Availability of data and materials}

All data generated or analysed during this study are included in this published article and its Additional files or deposited online. The mass spectrometry proteomics data have been deposited in the ProteomeXchange Consortium via the PRIDE [85] partner repository with the dataset identifier PXD019608. Phylogenetic datasets are available on the figshare repository (DOl:https://doi.org/10.6084/m9.figshare.12097692) [87].

\section{Ethics approval and consent to participate}

Not applicable

\section{Competing interests}

The authors declare that they have no competing interests.

\section{Author details}

'Department of Parasitology, Faculty of Science, Charles University, BIOCEV, Průmyslová 595, 25250 Vestec, Czech Republic. ${ }^{2}$ Department of Cell and Molecular Biology, Science for Life Laboratory, Uppsala University, SE-75123 Uppsala, Sweden.

Received: 16 December 2019 Accepted: 18 June 2020 Published online: 30 June 2020

\section{References}

1. Quenault T, Lithgow T, Traven A. PUF proteins: repression, activation and mRNA localization. Trends Cell Biol. 2011;21:104-12..

2. Qiu C, McCann KL, Wine RN, Baserga SJ, Hall TMT. A divergent Pumilio repeat protein family for pre-rRNA processing and mRNA localization. Proc Natl Acad Sci. 2014;111:18554-9.

3. Thomson E, Rappsilber J, Tollervey D. Nop9 is an RNA binding protein present in pre-40S ribosomes and required for 18S rRNA synthesis in yeast. Rna. 2007;13:2165-74.

4. Zhang B, Gallegos M, Puoti A, Durkin E, Fields S, Kimble J, et al. A conserved RNA-binding protein that regulates sexual fates in the $C$ elegans hermaphrodite germ line. Nature. 1997;390:477-84.
5. Barker DD, Wang C, Moore J, Dickinson LK, Lehmann R. Pumilio is essential for function but not for distribution of the Drosophila abdominal determinant Nanos. Genes Dev. 1992;6:2312-26.

6. Miller MT, Higgin JJ, Hall TM. Basis of altered RNA-binding specificity by PUF proteins revealed by crystal structures of yeast Puf $4 p$. Nat Struct Mol Biol. 2008;15:397-402.

7. Wang Y, Opperman L, Wickens M, Hall TM. Structural basis for specific recognition of multiple mRNA targets by a PUF regulatory protein. Proc Natl Acad Sci U S A. 2009;106:20186-91.

8. Hogan GJ, Brown PO, Herschlag D. Evolutionary conservation and diversification of Puf RNA binding proteins and their mRNA targets. PLoS Biol. 2015;13:e1002307.

9. Campbell ZT, Valley CT, Wickens M. A protein-RNA specificity code enables targeted activation of an endogenous human transcript. Nat Struct Mol Biol. 2014;21:732-8.

10. Liang X, Hart KJ, Dong G, Siddiqui FA, Sebastian A, Li X, et al. Puf3 participates in ribosomal biogenesis in malaria parasites. J Cell Sci. 2018;131: jcs212597.

11. Zhang C, Muench DG. A nucleolar PUF RNA-binding protein with specificity for a unique RNA sequence. J Biol Chem. 2015;290:30108-18.

12. Crittenden SL, Bernstein DS, Bachorik JL, Thompson BE, Gallegos M Petcherski AG, et al. A conserved RNA-binding protein controls germline stem cells in Caenorhabditis elegans. Nature. 2002;417:660-3.

13. Wang X, McLachlan J, Zamore PD, Hall TM. Modular recognition of RNA by a human pumilio-homology domain. Cell. 2002;110:501-12.

14. Blewett NH, Goldstrohm AC. A eukaryotic translation initiation factor $4 \mathrm{E}-$ binding protein promotes mRNA decapping and is required for PUF repression. Mol Cell Biol. 2012;32:4181-94.

15. Nyikó T, Auber A, Bucher E. Functional and molecular characterization of the conserved Arabidopsis PUMILIO protein, APUM9. Plant Mol Biol. 2019; 100:199-214.

16. Goldstrohm AC, Hook BA, Seay DJ, Wickens M. PUF proteins bind Pop2p to regulate messenger RNAs. Nat Struct Mol Biol. 2006;13:533-9.

17. Suh N, Crittenden SL, Goldstrohm A, Hook B, Thompson B, Wickens M, et al. FBF and its dual control of gld-1 expression in the Caenorhabditis elegans germline. Genetics. 2009;181:1249-60.

18. Garcia-Rodriguez LJ, Gay AC, Pon LA. Puf3p, a Pumilio family RNA binding protein, localizes to mitochondria and regulates mitochondrial biogenesis and motility in budding yeast. J Cell Biol. 2007;176:197-207.

19. Gerber AP, Herschlag D, Brown PO. Extensive association of functionally and cytotopically related mRNAs with Puf family RNA-binding proteins in yeast. PLoSBiol. 2004;2:E79.

20. Pederson T. The nucleolus. Cold Spring Harb Perspect Biol. 2011;3:1-15.

21. Zhang J, McCann KL, Qiu C, Gonzalez LE, Baserga SJ, Hall TMT. Nop9 is a PUF-like protein that prevents premature cleavage to correctly process pre18 r rRNA. Nat Commun. 2016;7:13085.

22. Wang B, Ye K. Nop9 binds the central pseudoknot region of $18 \mathrm{~S}$ rRNA. Nucleic Acids Res. 2017;45:gkw1323.

23. Li Z, Lee I, Moradi E, Hung NJ, Johnson AW, Marcotte EM. Rational extension of the ribosome biogenesis pathway using network-guided genetics. PLoS Biol. 2009;7.

24. Wickens M, Bernstein DS, Kimble J, Parker R. A PUF family portrait: 3'UTR regulation as a way of life. Trends Genet. 2002;18:150-7.

25. Tam PPC, Barrette-Ng $\mathbb{H}$, Simon DM, Tam MWC, Ang AL, Muench DG. The Puf family of RNA-binding proteins in plants: phylogeny, structural modeling, activity and subcellular localization. BMC Plant Biol. 2010;10.

26. Galgano A, Forrer M, Jaskiewicz L, Kanitz A, Zavolan M, Gerber AP. Comparative analysis of mRNA targets for human PUF-family proteins suggests extensive interaction with the miRNA regulatory system. PLoS One. 2008;3:e3164

27. O'Malley MA, Leger MM, Wideman JG, Ruiz-Trillo I. Concepts of the last eukaryotic common ancestor. Nat Ecol Evol. 2019;3:338-44.

28. Adl SM, Bass D, Lane CE, Lukeš J, Schoch CL, Smirnov A, et al. Revisions to the classification, nomenclature, and diversity of eukaryotes. J Eukaryot Microbiol. 2019;66:4-119.

29. Roger AJ, Muñoz-Gómez SA, Kamikawa R. The origin and diversification of mitochondria. Curr Biol. 2017;27:R1177-92.

30. Adam RD. Biology of Giardia lamblia. Clin Microbiol Rev. 2001;14:447-75.

31. Jiménez-García LF. The nucleolus of Giardia lamblia. MOJ Anat Physiol. 2017; $3: 41-3$. 
32. Elmendorf HG, Singer SM, Nash TE. The abundance of sterile transcripts in Giardia lamblia. Nucleic Acids Res. 2001;29:4674-83.

33. Nixon JEJ, Wang A, Morrison HG, MCArthur AG, Sogin ML, Loftus BJ, et al. A spliceosomal intron in Giardia lamblia. Proc Natl Acad Sci U S A. 2002;99: 3701-5.

34. Kamikawa R, Inagaki $Y$, Tokoro M, Roger AJ, Hashimoto T. Split introns in the genome of Giardia intestinalis are excised by spliceosome-mediated transsplicing. Curr Biol. 2011;21:311-5.

35. Li L, Wang CC. Capped mRNA with a single nucleotide leader is optimally translated in a primitive eukaryote, Giardia lamblia. J Biol Chem. 2004;279: 14656-64.

36. Hausmann S, Altura MA, Witmer M, Singer SM, Elmendorf HG, Shuman S. Yeast-like mRNA capping apparatus in Giardia lamblia. J Biol Chem. 2005; 280:12077-86.

37. Williams CW, Elmendorf HG. Identification and analysis of the RNA degrading complexes and machinery of Giardia lamblia using an in silico approach. BMC Genomics. 2011;12:586.

38. Frickey T, Lupas A. CLANS: a Java application for visualizing protein families based on pairwise similarity. Bioinformatics. 2004;20:3702-4

39. Almagro Armenteros JJ, Sønderby CK, Sønderby SK, Nielsen H, Winther O. DeepLoc: prediction of protein subcellular localization using deep learning Bioinformatics. 2017;33:3387-95.

40. Dolezal P, Smíd O, Rada P, Zubácová Z, Bursać D, Suták R, et al. Giardia mitosomes and trichomonad hydrogenosomes share a common mode of protein targeting. Proc Natl Acad Sci U S A. 2005;102:10924-9.

41. Martincová E, Voleman L, Pyrih J, Žárský V, Vondráčková P, Kolísko M, et al. Probing the biology of Giardia intestinalis mitosomes using in vivo enzymatic tagging. Mol Cell Biol. 2015;35:2864-74.

42. Hall TMT. Expanding the RNA-recognition code of PUF proteins. Nat Struct Mol Biol. 2014;21:653-5.

43. Jarmoskaite I, Denny SK, Vaidyanathan PP, Becker WR, Andreasson JOL, Layton CJ, et al. A quantitative and predictive model for RNA binding by human Pumilio proteins. Mol Cell. 2019;74:966-81 e18.

44. Morrison HG, McArthur AG, Gillin FD, Aley SB, Adam RD, Olsen GJ, et al. Genomic minimalism in the early diverging intestinal parasite Giardia lamblia. Science. 2007;317:1921-6.

45. Grant CE, Bailey TL, Noble WS. FIMO: scanning for occurrences of a given motif. Bioinformatics. 2011;27:1017-8.

46. Kershner AM, Kimble J. Genome-wide analysis of mRNA targets for Caenorhabditis elegans FBF, a conserved stem cell regulator. Proc Natl Acad Sci U S A. 2010;107:3936-41.

47. Yang YT, Ting YH, Liang KJ, Lo KY. The roles of Puf6 and Loc1 in $60 \mathrm{~S}$ biogenesis are interdependent, and both are required for efficient accommodation of Rpl43. J Biol Chem. 2016;291:19312-23.

48. Gu W, Deng Y, Zenklusen D, Singer RH. A new yeast PUF family protein, Puf6p, represses ASH1 mRNA translation and is required for its localization. Genes Dev. 2004;18:1452-65.

49. Zaremba-Niedzwiedzka K, Caceres EF, Saw JH, Di B, Juzokaite L, Vancaester $E$, et al. Asgard archaea illuminate the origin of eukaryotic cellular complexity. Nature. 2017;541:353-8.

50. Qiu C, Dutcher RC, Porter DF, Arava Y, Wickens M, Hall TMT. Distinct RNAbinding modules in a single PUF protein cooperate to determine RNA specificity. Nucleic Acids Res. 2019;47:8770-84.

51. Tatusov RL, Fedorova ND, Jackson JD, Jacobs AR, Kiryutin B, Koonin E V., et al. The COG database: an updated vesion includes eukaryotes. BMC Bioinformatics. 2003:4:1-14.

52. Vosseberg J, van Hooff JJE, Marcet-Houben M, van Vlimmeren A, van Wijk LM, Gabaldón T, et al. Timing the origin of eukaryotic cellular complexity with ancient duplications. bioRxiv. 2019::823484:1-18.

53. Ankarklev J, Jerlström-Hultqvist J, Ringqvist E, Troell K, Svärd SG. Behind the smile: cell biology and disease mechanisms of Giardia species. Nat Rev Microbiol. 2010;8:413-22

54. Prucca CG, Slavin I, Quiroga R, Elías EV, Rivero FD, Saura A, et al. Antigenic variation in Giardia lamblia is regulated by RNA interference. Nature. 2008; 456:750-4.

55. Andersen JS, Lyon CE, Fox AH, Leung AKL, Lam YW, Steen H, et al. Directed proteomic analysis of the human nucleolus. Curr Biol. 2002;12:1-11.

56. Narcisi EM, Glover CVC, Fechheimer M. Fibrillarin, a conserved pre-ribosomal RNA processing protein of Giardia. J. Eukaryot. Microbiol. 1998;45:105-11.
57. Jiménez-García LF, Zavala G, Chávez-Munguía B, Ramos-Godínez M d P, LópezVelázquez G, Segura-Valdez M d L, et al. Identification of nucleoli in the early branching protist Giardia duodenalis. Int J Parasitol. 2008;38:1297-304.

58. Tian XF, Yang ZH, Shen H, Adam RD, Lu SQ. Identification of the nucleoli of Giardia lamblia with TEM and CFM. Parasitol Res. 2010;106:789-93.

59. Li L, Wang CC. Identification in the ancient protist Giardia lamblia of two eukaryotic translation initiation factor $4 \mathrm{E}$ homologues with distinctive functions. Eukaryot Cell. 2005;4:948-59.

60. Saraiya AA, Wang CC. snoRNA, a novel precursor of microRNA in Giardia lamblia. PLOS Pathog. 2008;4:e1000224.

61. Lamanna AC, Karbsteina K. Nob1 binds the single-stranded cleavage site D at the 3 '-end of 18 S rRNA with its PIN domain. Proc Natl Acad Sci U S A. 2009:106:14259-64.

62. Teodorovic S, Walls CD, Elmendorf HG. Bidirectional transcription is an inherent feature of Giardia lamblia promoters and contributes to an abundance of sterile antisense transcripts throughout the genome. Nucleic Acids Res. 2007;35:2544-53.

63. Vanacova S, Liston DR, Tachezy J, Johnson PJ. Molecular biology of the amitochondriate parasites, Giardia intestinalis, Entamoeba histolytica and Trichomonas vaginalis. Int J Parasitol. 2003;33:235-55.

64. Filipovska A, Razif MF, Nygard KK, Rackham O. A universal code for RNA recognition by PUF proteins. Nat Chem Biol. 2011;7:425-7.

65. Burki F, Roger AJ, Brown MW, Simpson AGB. The new tree of eukaryotes. Trends Ecol Evol. 2020;35:43-55.

66. Zimmermann L, Stephens A, Nam SZ, Rau D, Kübler J, Lozajic M, et al. A Completely Reimplemented MPI Bioinformatics Toolkit with a New HHpred Server at its Core. J Mol Biol. 2018;430:2237-43.

67. Bailey TL, Johnson J, Grant CE, Noble WS. The MEME suite. Nucleic Acids Res. 2015;43:W39-49.

68. Aurrecoechea C, Barreto A, Basenko EY, Brestelli J, Brunk BP, Cade S, et al. EuPathDB: the eukaryotic pathogen genomics database resource. Nucleic Acids Res. 2017:45:D581-91.

69. Quast C, Pruesse E, Yilmaz P, Gerken J, Schweer T, Yarza P, et al. The SILVA ribosomal RNA gene database project: Improved data processing and webbased tools. Nucleic Acids Res. 2013;41:D590-6.

70. Katoh K, Standley DM. MAFFT multiple sequence alignment software version 7: improvements in performance and usability. Mol Biol Evol. 2013; 30:772-80.

71. Waterhouse AM, Procter JB, Martin DMA, Clamp M, Barton GJ. Jalview version 2-a multiple sequence alignment editor and analysis workbench. Bioinformatics. 2009;25:1189-91.

72. Capella-Gutiérrez S, Silla-Martínez JM, Gabaldón T trimAl: a tool for automated alignment trimming in large-scale phylogenetic analyses Bioinformatics 2009;25:1972-1973.

73. Price MN, Dehal PS, Arkin AP. FastTree 2 - approximately maximumlikelihood trees for large alignments. PLoS One. 2010;5:e9490.

74. Minh BQ, Schmidt HA, Chernomor O, Schrempf D, Woodhams MD, von Haeseler A, et al. IQ-TREE 2: new models and efficient methods for phylogenetic inference in the genomic era. Mol Biol Evol. 2020.

75. Hoang DT, Chernomor O, von Haeseler A, Minh BQ, Vinh LS. UFBoot2: improving the ultrafast bootstrap approximation. Mol Biol Evol. 2018;35: $518-22$

76. Lemoine F, Domelevo Entfellner JB, Wilkinson E, Correia D, Dávila Felipe M, De Oliveira T, et al. Renewing Felsenstein's phylogenetic bootstrap in the era of big data. Nature. 2018:556:452-6.

77. Kalyaanamoorthy S, Minh BQ, Wong TKF, von Haeseler A, Jermiin LS. ModelFinder: fast model selection for accurate phylogenetic estimates. Nat Methods. 2017;14:587-9.

78. Wang H-C, Minh BQ, Susko E, Roger AJ. Modeling site heterogeneity with posterior mean site frequency profiles accelerates accurate phylogenomic estimation. Syst Biol. 2018;67:216-35.

79. Keister DB. Axenic culture of Giardia lamblia in TYI-S-33 medium supplemented with bile. Trans R Soc Trop Med Hyg. 1983;77:487-8.

80. Pyrihová E, Motyčková A, Voleman L, Wandyszewska N, Fišer R, Seydlová G, et al. A single Tim translocase in the mitosomes of Giardia intestinalis illustrates convergence of protein import machines in anaerobic eukaryotes. Genome Biol Evol. 2018;10:2813-22.

81. Masuda T, Tomita M, Ishihama Y. Phase transfer surfactant-aided trypsin digestion for membrane proteome analysis. J Proteome Res. 2008;7:731-40.

82. Hebert AS, Richards AL, Bailey DJ, Ulbrich A, Coughlin EE, Westphall MS, et al. The one hour yeast proteome. Mol Cell Proteomics. 2014;13:339-47. 
83. Cox J, Hein MY, Luber CA, Paron I, Nagaraj N, Mann M. Accurate proteomewide label-free quantification by delayed normalization and maximal peptide ratio extraction, termed MaxLFQ. Mol Cell Proteomics. 2014;13: 2513-26.

84. Tyanova S, Temu T, Sinitcyn P, Carlson A, Hein MY, Geiger T, et al. The Perseus computational platform for comprehensive analysis of (prote) omics data. Nat Methods. 2016;13:731-40.

85. Vizcaíno JA, Csordas A, del-Toro N, Dianes JA, Griss J, Lavidas I, et al. 2016 update of the PRIDE database and its related tools. Nucleic Acids Res. 2016; 44:11033-11033.

86. Voleman L, Najdrová V, Ástvaldsson Á, Tưmová P, Einarsson E, Švindrych Z, et al. Giardia intestinalis mitosomes undergo synchronized fission but not fusion and are constitutively associated with the endoplasmic reticulum. BMC Biol. 2017;15:27.

87. : Najdrova V, Stairs CW, Vinopalová M, Voleman L, Dolezal P. The evolution of Puf superfamily proteins for rRNA maturation and mRNA translational regulation across the tree of eukaryotes. Supporting datasets. 2020. Figshare. DOI: https://doi.org/10.6084/m9.figshare.12097692.

\section{Publisher's Note}

Springer Nature remains neutral with regard to jurisdictional claims in published maps and institutional affiliations.

Ready to submit your research? Choose BMC and benefit from:

- fast, convenient online submission

- thorough peer review by experienced researchers in your field

- rapid publication on acceptance

- support for research data, including large and complex data types

- gold Open Access which fosters wider collaboration and increased citations

- maximum visibility for your research: over $100 \mathrm{M}$ website views per year

At BMC, research is always in progress.

Learn more biomedcentral.com/submissions 